\title{
A Protocol for Factor Identification
}

Kuntara Pukthuanthong, Richard Roll, and Avanidhar Subrahmanyam

June 23, 2018

\begin{abstract}
We propose a protocol for identifying genuine risk factors. A genuine risk factor must be related to the covariance matrix of returns, must be priced in the cross-section of returns, and should yield a reward-to-risk ratio that is reasonable enough to be consistent with risk pricing. A market factor, a profitability factor, and traded versions of macroeconomic factors pass our protocol, but many characteristic-based factors do not. Several of the underlying characteristics, however, do command premiums in the cross-section.
\end{abstract}

Contacts

\begin{tabular}{|c|c|c|c|}
\hline & Pukthuanthong & Roll & Subrahmanyam \\
\hline Voice: & $1-619-807-6124$ & $1-626-395-3890$ & $1-310-825-5355$ \\
\hline E-mail: & pukthuanthongk@missouri.edu & rroll@caltech.edu & asubrahm@anderson.ucla.edu \\
\hline Address: & $\begin{array}{l}\text { Department of Finance } \\
\text { University of Missouri } \\
\text { Columbia, MO } 65211\end{array}$ & $\begin{array}{c}\text { California Institute of } \\
\text { Technology } \\
\text { Pasadena, CA 91125 }\end{array}$ & $\begin{array}{c}\text { Anderson School } \\
\text { UCLA } \\
\text { Los Angeles, CA 90095-1481 }\end{array}$ \\
\hline
\end{tabular}

Acknowledgements

For insightful and constructive comments, we thank two anonymous referees, Andrew Karolyi (the editor), Dave Berger, Michael Brennan, Stephen Brown, Daniel Carvalho, Eric de Bodt, Wayne Ferson, Stefano Gubellinni, Campbell Harvey, Yan Liu, Peter Pope, Stephen Ross, Eduardo Schwartz, Kenneth Singleton, Ivo Welch, Russ Wermers and seminar participants at the 2012 Inquire UK conference, the 2013 Southwestern Finance Association annual meeting, the 2013 UCLA Brown Bag Seminar, the 2013 Rotman ICPM Seminar, the 2013 Australian Finance Conference, the 2014 Asian Finance Association Conference in Bali, the 2016 Dauphine-Amundi Conference in Paris, the 2016 Behavioral Finance Conference at the University of Miami, the 2016 Q-Group Conference in Phoenix, and the 2017 UBS/Maryland Conference in New York. We owe a great debt of gratitude to Olivier Ledoit for generous and unstinting guidance. We are thankful for financial support from Inquire UK, the Dauphine-Amundi Chair in Asset Management, the Rotman International Centre for Pension Management (ICPM) at the University of Toronto, and for the Q-Group's 2016 Jack Treynor Award. 


\title{
A Protocol for Factor Identification
}

\begin{abstract}
We propose a protocol for identifying risk factors. A genuine risk factor must be related to the covariance matrix of returns, must be priced in the cross-section of returns, and should yield a reward-to-risk ratio that is reasonable enough to be consistent with risk pricing. A market factor, a profitability factor, and traded versions of macroeconomic factors pass our protocol, but many characteristic-based factors do not. Several of the underlying characteristics, however, do command material premiums in the cross-section.
\end{abstract}


A factor model can be expressed as

$$
\mathbf{R}_{\mathbf{t}}=\mathbf{E}_{\mathrm{t}-1}\left(\mathbf{R}_{\mathrm{t}}\right)++\boldsymbol{\beta}_{\mathrm{t}-1} \mathbf{f}_{\mathrm{t}}+\boldsymbol{\gamma}_{\mathrm{t}-1} \mathbf{g}_{\mathrm{t}}+\boldsymbol{\varepsilon}_{\mathrm{t}}
$$

where $\mathbf{R}$ is an column vector of securities' returns in period t. ${ }^{1}$ Let $\mathrm{N}$ denote the number of securities. Assume that stochastic factors $\mathbf{f}$ command risk premiums and stochastic factors $\mathbf{g}$ do not. Given $\mathrm{K}$ risk factors, $\mathbf{f}$ is a $\mathrm{KX} 1$ mean zero column vector; the true risk factor loadings are in a matrix, $\boldsymbol{\beta}$, with $\mathrm{N}$ rows and $\mathrm{K}$ columns. Similarly, if there are $\mathrm{J}$ unpriced factors, $\mathbf{g}$ is a JX1 mean zero column vector and the associated loadings, $\gamma$, is a matrix with $\mathrm{N}$ rows and $\mathrm{J}$ columns. Finally, $\boldsymbol{\varepsilon}$ is an idiosyncratic NX1 mean zero column vector whose covariance matrix is diagonal. Notice that the loadings of both the true risk factors and the unpriced factors have time subscripts t- 1 to allow for time variation. The loadings are assumed to be known one period in advance of the returns.

The expected returns as of $\mathrm{t}-1$ conform to their own linear cross-sectional relation: ${ }^{2}$

$$
E_{t-1}\left(R_{t}\right)=R_{F, t-1}+\beta_{t-1} \lambda_{t-1}
$$

where the first term on the right is an NX1 column vector with the riskless rate at the beginning of the period in every position, $\lambda$ is a possibly time-varying $\mathrm{KX} 1$ column vector of non-zero risk premiums corresponding to factor class $\mathbf{f} .^{3}$ This implies that the factor set $\mathbf{g}$ is not priced in the crosssection of assets.

Empirically, how should one determine whether a particular candidate factor is in the set $\mathbf{f}$, the set $\mathbf{g}$, or neither of these sets? The job of any such procedure for factor identification should be to ascertain whether a particular factor candidate is in the $\mathbf{f}$ class and hence is unpredictable, is related

\footnotetext{
1 Hereafter, bold face indicates a vector or a matrix.

${ }^{2}$ See Connor and Korajczyk (1988) for an equilibrium version of this relation and Ross (1976) for an approximate version.

3 The arbitrage pricing theory represented by (2) holds exactly in an economy with infinitely many assets, and approximately otherwise.
} 
to systematic volatility and has an associated risk premium; or is in the $\mathbf{g}$ class and hence is unpredictable, is related to volatility but does not earn a risk premium or is neither priced nor related to asset volatility. A principal goal of our paper is to present a protocol for identifying whether a particular proposed factor is indeed a priced risk factor, i.e., belongs to class $\mathbf{f}$.

Note that Eq. (2) holds in a market where arbitrage is perfect and assets are not mispriced because of behavioral biases and arbitrage constraints. If asset mispricing is allowed, then deviations from Eq. (2) are permissible, and such deviation will be associated with "characteristics" that proxy for investor biases. Indeed, numerous factor candidates and firm-specific return predictors (characteristics) have been proposed in a voluminous literature. For example, Lewellen, Nagel, and Shanken (2010) list several of the most prominent predictor candidates in their opening paragraph and note that although they explain some empirical regularities, they have "...little in common economically with each other" (p. 175.) Subrahmanyam (2010) surveys more than fifty characteristics that various papers contend to be cross-sectionally related to mean returns. McLean and Pontiff (2016) examine 95 characteristics that were claimed in previous papers to explain returns cross-sectionally but find that predictability declines after publication. Lewellen (2015) finds strong predictive power of actual returns using 15 firm characteristics. Harvey, Liu, and Zhu (2016) enumerate 316 "factor" candidates suggested in 313 papers and suggest that any newly proposed factor should have to pass a much higher hurdle for statistical significance than the level habitually used in the literature, simply because of the extensive data mining. However, they do not attempt to relate the "factors" to the covariance matrix of returns, and do not draw a sharp distinction between firm-specific return predictors (characteristics) and priced factors. Green, Hand, and Zhang (2013) identify 330 firm characteristics and Green, Hand, and Zhang (2017) test whether 100 of them are priced (i.e., are associated with risk premiums.) They find that only 24 
characteristics are priced with an absolute t-statistic $\geq 3.0$.

Something needs to be done when more than 300 candidates have been suggested in the factor literature, and when there seems to be some confusion between priced "factors" and predictor "characteristics." New return predictors seem to be proposed in every issue of the major finance journals, adding to the existing ones, but there is no well-accepted process for determining their qualities. In addition, sometimes characteristic predictors are converted to their factor counterparts by computing the return differential across long-short decile portfolios formed based on the extreme values of the characteristics (Fama and French, 1993, 2008). At this point, there has been no protocol proposed in the literature to separately classify priced factors and non-priced factors. We need a process to evaluate them and to assess each additional predictor that will inevitably be nominated in the future.

We also note that there are few topics in finance, arguably none, that are more important than factor identification; factors are the main principal determinants of investment performance and risk. Indeed, the comparative values of well-diversified portfolios are determined almost completely by their factor exposures. Whether investors know it or not, every diversified portfolio is absolutely in thrall to factor drivers. Moreover, there seem to be more than one of them.

The multiplicity of factors is strongly suggested by two striking empirical regularities about portfolios. First, even really well-diversified portfolios are quite volatile. The volatility of a large positively-weighted portfolio is often around half as large as the average volatility of its constituents. For example, during the decade from 2001 through 2010, the monthly total return on the S\&P 500 had an annualized volatility (standard deviation) of 16.3\%. Over the same period, the average volatility for the S\&P's constituents was $36.1 \%$. 
Second, although well-diversified portfolios are highly correlated within the same asset class, they are much less correlated across classes; e.g., across bond vs. equities vs. commodities or across countries or across industry sectors. From 2001 through 2010, the monthly total return correlation between the S\&P 500 and Barclay's Bond Aggregate Index was -0.0426 . The return correlations between these two indexes and the Goldman Sachs Commodity index were 0.266 and 0.0113, respectively. Similarly modest correlations are typical between real estate assets and assets in other classes. ${ }^{4}$

The first empirical fact indicates the existence of at least one common underlying systematic influence, (or "risk driver" or "factor") that limit diversification within an asset class; otherwise diversified portfolios would have much smaller volatilities. The second fact implies the presence of multiple systematic factors across assets; otherwise diversified portfolios would be more correlated across asset classes, countries, and sectors.

Almost all academics and probably the vast majority of finance professionals now recognize that pervasive factors are among the main drivers of observed returns, but there is considerable disagreement about the identities of factors and even about whether they represent risks, anomalies, or something else.

Theory suggests that a true risk factor (in the class $\mathbf{f}$ in Eq. (1)) has three fundamental attributes:

(1) It varies unpredictably in a time series sense

(2) Its variations induce changes in asset prices

(3) It is associated with a risk premium.

Quasi-factors (in the set $\mathbf{g}$ ) influence the returns of few securities and are unpriced in aggregate. A

\footnotetext{
${ }^{4}$ Cotter and Roll (2015) report that real estate investment trusts have rather low betas against the S\&P 500.
} 
factor of this type possesses two attributes:

(1) It varies unpredictably in a time series sense

(2) Its variations do not affect expected returns.

Characteristics are sometimes associated with factors, but a characteristic

(1) Is known in advance

(2) Might be cross-sectionally related to the expected returns of some assets, and/or

(3) Might be cross-sectionally related to the loadings on true risk factors or the loadings on quasi factors.

Our main goal is to popularize a process to identify factors that will be broadly acceptable to both scholars and practitioners. We believe this is the first attempt to suggest a complete normative process for dealing with one of the most fundamental questions in finance: how to identify systematic risk factors that are reliably associated with expected returns. Our protocol has the potential to identify factors associated with risk premiums or true factors, but also factors that move some returns but do not have associated risk premiums, and characteristics that are associated with systematic return differences but are not related to risk. Characteristics that are reliably associated with returns but not risks are perhaps the most interesting of all, since they offer potential profit opportunities. ${ }^{5}$

The protocol is composed of a sequence of steps to check necessary conditions and one final step that examines a sufficient condition. From Eq. (1) (suppressing time subscripts and assuming orthogonal factors), we have the familiar relation

$$
\operatorname{cov}(\mathbf{R})=\beta \beta^{\prime} \operatorname{var}(\mathbf{f})+\gamma \gamma^{\prime} \operatorname{var}(\mathbf{g})+\left(\gamma \boldsymbol{\beta}^{\prime}+\beta \gamma^{\prime}\right) \operatorname{cov}(\mathbf{f}, \mathbf{g})+\mathbf{E}\left(\varepsilon \varepsilon^{\prime}\right),
$$

\footnotetext{
${ }^{5}$ Engelberg, McLean, and Pontiff (2017) show that the profitability from anomalies is higher around earnings announcement days after controlling for risk, which indicates that the anomalies in fact capture mispricing. Linnainmaa and Roberts (2017) argue that several recently discovered return predictors ("characteristics") might be spurious as they do not survive in the data from earlier time periods.
} 
where $\mathbf{E}\left(\boldsymbol{\varepsilon} \varepsilon^{\prime}\right)$ is diagonal. Eq. (3) implies that a necessary condition for any factor candidate is that it must be related to the covariance matrix of returns. Although this condition for the factor existence (correlation with the assets in question) is well known and used to various extents in much of the empirical work, our protocol presents a more systematic treatment of the subject. Note that this necessary condition does not distinguish between pervasive priced factors (those with risk premiums) and unpriced factors. Our sufficient condition tests provide for this distinction, by estimating associate risk premiums, if any.

We note that our paper is not aimed at testing a particular asset-pricing model, in contrast to studies by Lewellen and Nagel (2006) and Campbell et al. (2017), both of which examine the validity of the "conditional" CAPM. For reasons mentioned above, we think that any single factor theory, albeit conditional, cannot explain why diversified portfolios in different asset classes are so weakly correlated.

A single stochastic discount factor (SDF) or a conditional mean/variance efficient portfolio, is always available to conditionally explain the cross-section of asset returns with a single exposure coefficient. ${ }^{6}$ There, however, is a mapping from the SDF to the factor model. To see this, let us suppress time subscripts and note that the Euler equation states the following:

$$
\mathrm{E}(\mathbf{R M}) \mathbf{1},
$$

where M is the SDF and $\mathbf{1}$ is the unit vector. Substituting for the factor model from (1), we have

$$
\mathrm{E}(\mathbf{R})=\mathrm{a}_{1}+\mathrm{a}_{2} \boldsymbol{\beta}+\mathrm{k},
$$

where $\mathrm{a}_{1}$ and $\mathrm{a}_{2}$ are constants and $\mathrm{k}=-\mathrm{E}(\boldsymbol{\varepsilon M}) / \mathrm{E}(\mathrm{M})$. Now, the linear form of Eq. (2) holds as long as $k=0,{ }^{7}$ for which it suffices that $\mathrm{E}(\boldsymbol{\varepsilon M})=0$. This is true from Eq. (1) as long as the SDF M is a

\footnotetext{
${ }^{6}$ As emphasized by Cochrane (2001) and Singleton (2006, chapter 8)

${ }^{7}$ With the additional observation that the risk-free rate is the reciprocal of the expected value of $\mathrm{M}$ (see, for example, Campbell and Cochrane, 2000, for details).
} 
linear function of the factors. Thus, multiple factors are a practical way to explain unconditional returns over any finite sample.

From a practical perspective, either of an investor or a financial econometrician, incomplete information, via the finite sample problem, is inevitable. Our aim is to popularize an identification procedure for risk and non-risk factors that is useful, though perhaps not theoretically pristine in the sense of being congruent with a SDF. We illustrate our protocol using popular factors, which are based on fundamentals-driven, or characteristics-driven, arguments.

\section{Related Research}

One related study is by Charoenrook and Conrad (2008) (hereafter CC.) Their approach is motivated by Section 6.3 in Cochrane (2001), which derives a relation between the conditional variance of a true factor and that factor's associated risk premium. CC notice an important implication; viz., that time variation in a factor candidate's volatility should be correlated positively with time variation in its expected return. Consequently, if (a) a proposed factor has significant intertemporal variation, (b) its mean return also has significant variation, and (c) the two are positively correlated, then the factor candidate satisfies a necessary condition to be proxying for a true underlying priced factor. As CC emphasize though, such an empirical finding is not a sufficient condition.

$\mathrm{CC}$ find empirically that several proposed factor candidates, including size, book/market, and a liquidity construct, satisfy the above necessary condition. Momentum ${ }^{8}$ does not. Momentum's estimated mean/volatility relation has the wrong sign. If this finding is upheld, it

\footnotetext{
${ }^{8}$ A factor candidate originally proposed by Carhart (1997).
} 
implies strongly that the momentum characteristic offers a free lunch, supposedly an arbitrage opportunity.

In the last section of their paper, $\mathrm{CC}$, motivated by the recognition that their empirical condition is only necessary, examine whether the risk premiums associated with size, book/market and liquidity are in a plausible range. They find that the Sharpe ratios of size and book/market are plausible, but the Sharpe ratio for liquidity is not. We are left in doubt as to which of these are priced factors. We note that although size, book/market and liquidity satisfy a necessary condition to be risk factors, a test of sufficiency would build on their work. Also, since time variation in risk premiums is required for the $\mathrm{CC}$ necessary condition, a method that identifies factor candidates with stable risk premiums or with statistically small variation would also be complementary to their work.

Another related and recent paper is Harvey and Liu (2016). They propose a bootstrap method to select among a large group of candidate factors. They ascertain the factor from a pool of candidates that yields the lowest intercept in a cross-sectional model. They then find a second factor that yields the lowest intercept from the base model that has the first successful factor. The process is repeated until no further factor passes a significant hurdle.

To illustrate, suppose they have seven candidate factors and the market factor is the first successful factor, so it is added to the model. Then, in the second round, HML is the best factor, so it is added to the model that already has the market factor. In the third round, suppose SMB is the best factor but it has an insignificant $\mathrm{p}$-value. They stop at this point and declare that the market and HML are the only significant factors.

Fama and French (2017) propose squared Sharpe ratios to select factors. But an anomaly can have a high Sharpe ratio and not be risk related. Both the Harvey and Liu (2016) and the Fama 
and French (2017) protocols are useful, but linking the candidate factors to the sample covariance matrix is an additional step that would advance their work.

Barillas and Shanken (2018) propose a Bayesian asset pricing test that allows comparison of all possible asset pricing models from subsets of given factors. Feng, Giglio and Xiu (2017) propose the combination of the double-selection LASSO method of Belloni et al. (2014) with twopass regressions such as Fama-MacBeth to systematically select the best control model out of the large set of factors, while explicitly taking into account that in any finite sample we cannot be sure to have selected the correct model. Applying the key principle that true factors have to be related to the covariance matrix, which these papers do not do, would again be a useful exercise that would supplement their work.

Our protocol identifies not only factors associated with risk premiums or true factors, but also factors that move some returns but do not have associated risk premiums, and factors or characteristics that are associated with systematic return differences but not risks. Factors or characteristics that are reliably associated with returns but not risks are perhaps the most interesting of all, since they offer potential profit opportunities. Although the papers mentioned above have the same goal as ours, they do not distinguish among these categories of factors.

\section{Factors and the Covariance Matrix}

A necessary condition for any empirically measurable candidate (like Fama and French's (1993) HML) to be a factor is that it be related to the principal components of the covariance matrix. This condition represents the motivation for the analysis in Moskowitz (2003), who checks it for three candidates, size, book/market, and momentum. Moskowitz finds that size satisfies the condition; it is related to covariation and its associated risk premium is positively associated with its volatility. 
Book/market is close to satisfying but momentum is not. This agrees with the results of CC discussed above in the case of momentum, and it more or less agrees with CC in the case of book/market.

Unfortunately, in our imperfect world, factor extraction from the covariance matrix faces a number of serious difficulties, including

a. It produces only estimates for linear combinations of the true underlying factors, not the factors themselves;

b. It is compromised by non-stationarity since there is no plausible reason why the number of factors or their relative importance should be constant through time ${ }^{9}$;

c. It includes true risk drivers, pervasive priced factors (or linear combinations thereof) along with unpriced factors.

Fortunately, there is a remedy, perhaps imperfect, for each of these conundrums. For (a), the linear combinations extracted by PCA could be related to other candidate factors, such as macroeconomic variables, through canonical correlation or a similar method. This wouldn't prove anything but it would at least give some reassurance or raise some serious doubt. For (b), PCAs could be estimated for subperiods or with models that accommodate non-stationarity. For (c), a second stage method as in Fama and MacBeth (1973) could be employed to distinguish priced factors from others. Needless to say, none of these cures is without its own problems. ${ }^{10}$

\footnotetext{
${ }^{9}$ Moreover, it seems that non-stationarity is an empirical fact. Moskowitz (2003) finds that “... significant time variation in the covariance structure of asset returns distorts the ability of these time-invariant factors (principal components extracted from the unconditional covariance matrix) to capture second moments, suggesting that unconditional factors miss important dynamics in return volatility," (p. 436).

${ }^{10}$ It is known that PCA will do a rotation that makes it seem that the first factor is more dominant than in the true underlying structure. Brown (1989) offers a remedy. However, this problem is not all that troubling for our protocol because we do not need to determine the true number of underlying factors, but merely that a factor candidate is related to some PCA extracted from the covariance matrix. Just one, the first PCA, is sufficient for a factor candidate to pass the necessary conditions.
} 


\section{What are the Underlying Factors?}

What exactly are the salient features of factors, the underlying risk drivers? Cochrane (2001) says

unequivocally, "The central and unfinished task of absolute asset pricing ${ }^{11}$ is to understand and measure the sources of aggregate or macroeconomic risk that drive asset prices." (p. xiv.) He particularly has in mind aggregate consumption as a driver and even goes so far as to say that “...the only consistent motivation for factor models is a belief that consumption data are unsatisfactory," (p. 170, emphasis in original.) In other words, if we only had adequate measures of aggregate consumption, we wouldn't need much else for risk modeling. The absence of adequate consumption data motivates the study of other indicators of macroeconomic activity, even hundreds of such indicators.

The underlying drivers cannot be the infrequently-published official numbers about macroeconomic variables because market prices move around much too rapidly. Instead, the drivers must be high-frequency changes in privately-held market perceptions of pervasive macroeconomic conditions. Perceptions could include (a) rational anticipations of change in macro conditions that are truly pervasive such as real output growth, real interest rates, inflation, energy, etc., and (b) behavior-driven pervasive shocks in confidence or risk perceptions such as panics, liquidity crises, etc.

To do a really good job, we must be able to identify and measure the pervasive factor perceptions and then to estimate factor sensitivities (betas) for every real asset. The first job is to identify and measure the factors. Existing literature has studied several alternative approaches. As discussed in the previous section, one approach relies on an entirely statistical method such as principal components or factor analysis, (e.g., Roll and Ross, 1980; Connor and Korajczyk, 1988.)

\footnotetext{
${ }^{11}$ As opposed to relative asset pricing such as comparing an option price to the underlying stock price.
} 
A second approach pre-specifies macroeconomic variables that seem likely to be pervasive and then pre-whitens the official numbers pertaining to such low frequency constructs as industrial production, inflation, and so on, (e.g., Chen, Roll and Ross, 1986.) Then there is the approach of relying on asset characteristics to develop proxies that are empirically related to average returns (e.g., Fama and French, 1993, Carhart, 1997.)

\section{Putting it All Together: Linking Proposed Factors to the Covariance Matrix}

Given the discussion above, we are ready to outline the first stage of our protocol for identifying factors. This stage identifies factors that move asset prices systematically but it does not distinguish between pervasive priced factors (with risk premiums) and unpriced ones. That crucial information is postponed to a later stage. Here are the recommended steps for this first stage.

First, collect a set of $\mathrm{N}$ equities for the factor candidates to explain. The test assets should belong to different industries and have enough heterogeneity so that the underlying risk premium associated factors can be detected.

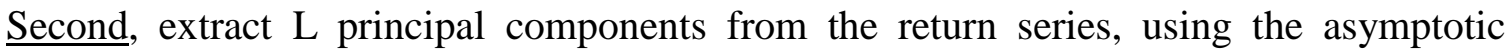
approach of Connor and Korajczyk (CK) (1988). With T time-series units up to time t, the procedure involves computing the TxT matrix $\boldsymbol{\Omega}_{\mathbf{t}}=(\mathbf{1} / \mathbf{T}) \mathbf{R} \mathbf{R}^{\prime}$, where $\mathbf{R}$ is the return vector. CK show that for large $\mathrm{N}$, analyzing the eigenvectors of $\boldsymbol{\Omega}_{\mathbf{t}}$ is asymptotically equivalent to factor analysis. The first L eigenvectors of $\boldsymbol{\Omega}_{\mathbf{t}}$ form the factor estimates. The cutoff point for $\mathrm{L}<\mathrm{N}$ should be designated in advance; for instance, L could be chosen so that the cumulative variance explained by the principal components is at least ninety percent. Note that since, in most finance applications, $\mathrm{N}>>\mathrm{T}$, the approach has the virtue of allowing us to work with the smaller-dimension TxT matrix $\boldsymbol{\Omega}_{\mathbf{t}}$, as opposed to the traditional $\mathrm{NxN}$ covariance matrix used for factor analysis. 
$\underline{\text { Third, }}$ collect a set of $\mathrm{K}$ factor candidates. These could be well known characteristics-based candidates such as size, book/market, momentum, or any of the 50 or so documented in Subrahmanyam (2010), or the 316 from Harvey et al. (2016), or any new candidate as yet to be suggested.

Fourth, compute canonical correlations between the factor candidates and the corresponding eigenvectors from the second step. To do this, first certain matrix computations are necessary. First, using the L eigenvectors from step \#2 and the $\mathrm{K}$ factor candidates from step \#3, calculate the covariance matrix over a period up to time $t, \mathbf{V}_{\mathbf{t}}(\mathbf{L}+\mathbf{K} \times \mathbf{L}+\mathbf{K})$. Next, from the covariance matrix $\mathbf{V}_{\mathbf{t}}$, in each period t, break out a sub-matrix, the cross-covariance matrix, which we denote $\mathbf{C}_{t}$. It has $\mathrm{K}$ rows and $\mathrm{L}$ columns (i.e., $\mathrm{K} \mathrm{x} \mathrm{L}$ ); the entry in the $\mathrm{i}^{\text {th }}$ row and $\mathrm{j}^{\text {th }}$ column being the covariance between factor candidate $\mathrm{i}$ and eigenvector $\mathrm{j}$. It will also be necessary to break out the covariance sub-matrix of the factor candidates, $\mathbf{V}_{\mathbf{f}, \mathbf{t}}(\mathbf{K} \times \mathbf{x})$ and the covariance submatrix of the real eigenvectors, $\mathbf{V}_{\mathrm{e}, \mathrm{t}}(\mathbf{L} \mathbf{x} \mathbf{L})$. These computations allow us to then find two weighting column vectors, $\lambda_{t}$ and $\kappa_{t}$, on the factor candidates and eigenvectors, respectively $\left(\lambda_{t}\right.$ has $\mathrm{K}$ rows and $\kappa_{t}$ has $\mathrm{L}$ rows) that maximize the correlation between the two weighted vectors. The covariance between the weighted averages of factor candidates and eigenvectors is $\lambda_{t}{ }^{\prime} C_{t} \kappa_{t}$, and their correlation is

$$
\rho=\frac{\lambda_{t}^{\prime} C_{t} \kappa_{t}}{\sqrt{\lambda_{t}^{\prime} V_{f, t} \lambda_{t} \kappa_{t}^{\prime} V_{e, t} \kappa_{t}}}
$$

The correlation is maximized over all choices of $\lambda_{t}$ and $\kappa_{t}$. It turns out that the maximum occurs when the weights satisfy $\lambda_{t}=V_{f, t}^{-1 / 2} h_{t}$ where $h_{t}$ is the eigenvector corresponding to the maximum eigenvalue in the matrix $V_{f, t}^{-1 / 2} C_{t} V_{e, t}^{-1} C_{t}^{\prime} V_{f, t}^{-1 / 2}$. The vector $\kappa_{t}$ is proportional to $h_{t}$. One then maximizes the correlation again, subject to the constraint that the new vectors are orthogonal 
to the old one, and so on. This way, there are $\min (\mathrm{L}, \mathrm{K})$ pairs of orthogonal canonical variables sorted from the highest correlation to the smallest. Each correlation can be transformed into a variable that is asymptotically distributed as Chi-Square under the null hypothesis that the true correlation is zero. ${ }^{12}$ This provides a method of testing whether the factor candidates as a group are conditionally related (on date t) to the covariance matrix of real returns (as represented by Eq. (3)). Also, by examining the relative sizes of the weightings in $\lambda_{t}$, one can obtain an insight into which factor candidates, if any, are more related to real return covariances. We describe the latter procedure in detail within our empirical application in Section 7.

The intuition behind the canonical correlation approach is straightforward. The true underlying drivers of real returns are undoubtedly changes in perceptions about macroeconomic variables (see Section 3 above). But the factor candidates and the eigenvectors need not be isomorphic to a particular macro variable. Instead, each candidate or eigenvector is some linear combination of all the pertinent macro variables. This is the well-known "rotation" problem in principal components or factor analysis. ${ }^{13}$ Consequently, the best we can hope for is that some linear combination of the factor candidates is strongly related to some different linear combination of the eigenvectors that represent the true factors in Eq. (1). Canonical correlation is intended for exactly this application.

Any factor candidate that does not display a significant (canonical) correlation with its associated best linear combination of eigenvectors can be rejected as a viable factor. It is not significantly associated with the covariance matrix of real asset returns.

\footnotetext{
12 See Anderson (1984, ch. 12) or Johnson and Wichern (2007).

13 The rotation problem is resolved by placing restrictions on the extracted factors. In principal components, the restriction is that successive factors explain the maximum amount of remaining variance. In factor analysis, restrictions are imposed on the factor covariance matrix; (e.g., it is diagonal or lower triangular.)
} 


\section{Putting it All Together: Testing for Whether a Risk Factor is Priced}

In principle, the sufficiency stage of ascertaining whether factor candidates command risk premiums is easy. We simply run a pooled cross-section/time series panel with real returns as dependent variables and betas on surviving factors as the explanatory variables, taking account of correlations across assets and time (Cf. Petersen, 2009). This should be done with individual real asset returns on the left side, not with portfolio returns, because portfolios might diversify away and thus mask relevant risk- or return-related features of individual assets. Diversification into portfolios can mask cross-sectional phenomena in individual assets that are unrelated to the portfolio grouping procedure. Roll (1977) argues that the portfolio formation process makes it difficult to reject the null hypothesis of no effect on security returns. Advocates of fundamental indexation (Arnott, Hsu and Moore, 2005) argue that high market value assets are overpriced and vice versa, but any portfolio grouping by an attribute other than market value itself could diversify away such mispricing, making it undetectable.

Second, test portfolios are typically organized by firm characteristics related to average returns, e.g., size and book-to-market. Sorting on characteristics that are known to predict returns helps generate a reasonable variation in average returns across test assets. However, Lewellen, Nagel, and Shanken (2010) point out sorting on characteristics also imparts a strong factor structure across test portfolios. Lewellen et al. (2010) show that even factors that are weakly correlated with the sorting characteristics would explain the differences in average returns across test portfolios regardless of the economic theories underlying the factors. They caution about the low dimensionality issue when portfolios are used, decreasing test power because there are fewer observations with portfolios than with individual assets. Lo and MacKinlay (1990) support this strand of the argument and show that, in contrast to Roll (1977), forming portfolio on 
characteristics makes it likely to reject the null hypothesis too often because of a "data snooping" bias.

Third, forming portfolios might mask cross-sectional relation between average returns and factor exposures ("betas"). To illustrate, the cross-sectional relation between expected returns and betas under the single-factor CAPM holds exactly if and only if the market index used for computing betas is on the mean/variance frontier of the individual asset universe. Errors from the beta/return line, either positive or negative, imply that the index is not on the frontier. But if the individual assets are grouped into portfolios sorted by portfolio beta and the individual errors are not related to beta, the analogous line fitted to portfolio returns and betas will display much smaller errors. This could lead to a mistaken inference that the index is on the efficient frontier.

Finally, the statistical significance and economic magnitudes of risk premiums could depend on the choice of test portfolios. For example, the Fama and French size and book-to-market risk factors are significantly priced when test portfolios are sorted based on corresponding characteristics, but they do not command significant risk premiums when test portfolios are sorted only based on momentum. Brennan, Chordia, and Subrahmanyam (1998) also show different results for different sets of portfolios depending on characteristics used to form such portfolios.

The preceding discussion indicates that a properly-specified regression analysis based on individual securities is more desirable than a portfolio approach to identify risk premiums. A variant of the panel approach of Petersen (2009) is standard in finance; it was originated by Fama and MacBeth (FM) (1973). The only real difficulty is that the regression betas, the factor loadings, are not known quantities and must be estimated. This implies a classic errors-in-variables (EIV) problem because the betas are the explanatory variables in each FM cross-section. Since the 
estimated betas inevitably contain measurement errors, the cross-sectional regressions have biased coefficients.

The error variances for individual assets are almost certainly greater than they are in betas estimate for portfolios, which explains why Fama and French (1992) use the latter. In our analysis, we adopt their procedure in using portfolios to obtain beta estimates, assigning portfolio betas to the constituent individual stocks, and then checking to see if the factor is priced via FM regressions. While this exercise is performed on the market factor in Fama and French (1992), it has not been performed consistently on other factors. Indeed, Fama and French (1993) do not perform this second stage exercise on individual securities for their SMB and HML factors.

To specifically address the EIV problem, we do a double sorting. First, we sort stocks based on size into ten portfolios; then, within each size decile, we sort stocks into ten portfolios by market beta. Next, we independently do the same double sorts but instead of sorting by market beta, we sort by HML beta. We do the same double sort for every factor that passes our necessary conditions. Then, we assign each of these portfolio betas to the stocks that the portfolio contains. Following this assignment procedure, we consider the significance of the betas in FM regressions.

As a final check following the FM regression, we propose that for a genuine risk factor, its reward-to-risk ratio must be within reasonable limits. To take an extreme example, if a candidate risk factor delivers a Sharpe ratio of three, it would be difficult to accept such a magnitude as indicative of a priced risk, given that Sharpe ratios for most well-diversified market indices are usually less than unity over periods of a decade or more (MacKinlay, 1995).

Thus, we propose an investigation of whether factor-based Sharpe ratios exceed a "reasonable bound." Our bound is the one proposed by MacKinlay (1995). He argues that based on the historical mean excess return and volatility of the CRSP value-weighted index, a reasonable 
annualized Sharpe ratio for a risk factor is 0.6 (corresponding for example, to an annualized excess return of $10 \%$ and a standard deviation of $16 \%$ ). We propose to test whether each individual proposed factor delivers a Sharpe ratio is statistically higher than the proposed MacKinlay bound.

\section{Simulation}

We perform a simulation exercise to assess whether our protocol reliably identifies risk factors that are priced cross-sectionally and explains covariation in asset returns. In the basic setup, we simulate five factors associated with risk premiums (f), three factors that do not command risk premiums (g), and five characteristics $(\mathrm{Z})$. Our simulations are all based on the Gaussian crosssectional or time series distributions.

We use the actual mean excess return and standard deviation of the value-weighted CRSP index to generate the time-series of the first priced factor $f_{1}$. The other four priced factors are generated so that they are mutually independent and independent of $\mathrm{f}_{1}$, from a distribution with the actual mean $(0.354 \%)$ and standard deviation $(2.897 \%)$ of a well-known factor, the Fama and French HML, over our sample period. Since factors g are unpriced, they each have a mean of zero. The standard deviation of each of the g's is set equal to that of HML. The five characteristics (Zs) represent firm-specific information, which varies across time; therefore, they are generated during each time period from the uniform distribution $[-0.1 \%, 0.1 \%]{ }^{14}$

We draw 5,000 data sets from the data generating process (DGP). For each data, we check both necessary and significant condition of our protocol from a pool of 13 factor candidates. To make our simulation more realistic, our candidate factors are noisy versions of $\mathrm{f}$ and $\mathrm{g}$. In particular, we add estimation errors (or noise) $\eta$ and $\imath$ to the factors $\mathrm{f}$ and $\mathrm{g}$ respectively and

\footnotetext{
${ }^{14}$ An alternative way to simulate characteristics would be to draw them from autocorrelated (e.g., autoregressive) processes for each firm. Since it is not immediately obvious how the autocorrelation should vary in the crosssection, we leave this exercise for future work.
} 
denote the candidate factors as $\mathrm{f}$ and $\mathrm{g}$. These errors have zero mean and a standard deviation that is parametrically varied in the simulations. ${ }^{15}$

More specifically, our DGP is based on the following equation:

$$
R_{t}=r_{f}+\beta f_{t}+\gamma g_{t}+k Z_{t}+\varepsilon_{t}
$$

where $R_{t}$ is a $\mathrm{N}$-asset vector of returns in period $\mathrm{t}, \mathrm{t}=1,2, \ldots, \mathrm{T}$. Further:

- $r_{f}$ is a time-invariant risk-free rate, set to $0.1 \%$

- $f_{t}=\left(\mathrm{f}_{1, t}, \mathrm{f}_{2, t}, \mathrm{f}_{3, t}, \mathrm{f}_{4, t}, \mathrm{f}_{5, t}\right)$ is a $5 \times 1$ vector of independent risk factors at time $\mathrm{t}$.

- $\mathrm{f}_{1, t}$ is normally distributed with the mean and standard deviation of Rm-Rf. $\mathrm{f}_{\mathrm{i}, t} \mathrm{i} \neq 1$ is also normally distributed, but with the mean and standard deviation of the HML factor.

- $\quad \beta$ is a $\mathrm{N} \times 5$ asset-specific factor loading matrix for the five factors that carry a risk premium. $\beta$ is independently drawn from $\mathrm{N}(1,1)$ for each firm.

- $g_{t}=\left(\mathrm{g}_{1, t}, \mathrm{~g}_{2, t}, \mathrm{~g}_{3, t}\right)$ is a $3 \mathrm{x} 1$ vector of independent unpriced factors at time t. $g_{\mathrm{i}, t}$ is normally distributed with a mean of zero and a standard deviation equal to that of HML where $\mathrm{i}=1,2,3$. The loadings on $\mathrm{g}$, i..e, $\gamma$, are drawn in a manner similar to $\beta$.

- $Z_{t}=\left(\mathrm{Z}_{1, t}, \mathrm{Z}_{2, t}, \mathrm{Z}_{3, t}, \mathrm{Z}_{4, t}, \mathrm{Z}_{5, t}\right)$ is a $\mathrm{N} \times 5$ matrix of characteristics at time $\mathrm{t}$. In particular, $\mathrm{Z}_{\mathrm{i}, t}$ is an $\mathrm{Nx} 1$ asset-specific vector of the $i^{\text {th }}$ characteristic. $\mathrm{Z}_{\mathrm{i}, t}$ is drawn at each time period $\mathrm{t}$ from a uniform distribution $[-0.1 \%, 0.1 \%]$.

- $\mathrm{k}=(1,1,1,-1,-1)$ is a $5 \times 1$ constant vector.

${ }^{15}$ We have tried other DGPs for returns. While they lead to broadly similar results, we desist from reporting results from other variants; on the rationale that this exercise might obscure the nature of our protocol. 
- $\varepsilon_{t}$ is an $\mathrm{Nx} 1$ vector of the firm-specific return components. It is assumed to follow a normal distribution with mean zero. The variance of this term is chosen such that for a “typical” stock whose betas equal their cross-sectional means, the $\mathrm{R}^{2}$ from a regression of individual stock returns on the factors equals $25 \% .^{16}$

Our factor candidates (FC) are denoted by

- $f_{i, t}=f_{i, t}+\eta_{i, t}$ is a noisy factor with measurement error $\eta_{i, t} \sim N(\mu=0, \sigma)$ where $\mathrm{i}=1,2,3,4,5$.

- $g_{i, t}=g_{i, t}+\iota_{i, t}$ is a noisy factor with measurement error $\iota_{i, t} \sim N(\mu=0, \sigma)$ where $\mathrm{i}=1,2,3$.

For each of the 5,000 drawn data sets, we apply the asymptotic approach of Connor and Korajczyk (1988) to extract 10 principal components from the simulated return series. We compute canonical correlations (i.e., $\rho$ ) between the 10 principal components and the 13 factor candidates, and test the significance of these canonical correlations by the Chi-square statistic described in the fourth step of Section 4 . We proceed only with statistically significant canonical correlations and test the significance of each factor candidate as follows: First, for each of the significant canonical correlations, the corresponding canonical variate, say $\mathrm{U}$, is constructed from a linear combination of PCs, whose coefficients come from the weighting vector $\kappa$ in the fourth step of Section 4. Second, we run a regression of $U$ on the all factor candidates. The t-statistics from the regression then present the significance level of each factor candidate. Any given factor candidate passes the necessary condition of our protocol if the mean t-statistic over all significant canonical correlations is significant at the $5 \%$ level or exceeds 1.96 .

\footnotetext{
16 This threshold corresponds to the higher end of the estimates in Campbell, Lettau, Malkiel, and Xu (2001) that use but a single (market) factor; however, our results are not particularly sensitive to the specific assumption.
} 
Next, we examine the sufficient condition for factor candidates that satisfy the necessary condition. Specifically, we ask if a factor is priced cross-sectionally. We perform a Fama-MacBeth (FM) regression as follows: first, each asset return is regressed on the time series of all factor candidates surviving the necessary condition. Second, the stock return is regressed crosssectionally on the factor loadings (or betas), resulting in a series of risk premium coefficients, say $v_{i t}$, for each surviving factor. The risk premium for a given factor is computed by averaging the $v_{i t}$ over T. We test for the significance of the risk premiums via their usual time-series t-statistics (i.e., the mean of each $v_{i t}$ times the square root of $\mathrm{T}$ divided by the standard deviation of $v_{i t}$ ).

We also control for asset-specific characteristics. Thus, in a variant of the FM regression above, instead of using factor loadings (or betas) alone in the sufficient condition, we also include the five characteristics $\mathrm{Z}$ in the DGP and compute the risk premiums for the surviving factors and the premiums for these characteristics. Table 1 presents the percentages that pass the necessary conditions and significance rate of each factor in the sufficient condition after 5,000 simulations. The number of stocks $\mathrm{N}$ ranges from 2000 to 6000 whereas the number of time periods $\mathrm{T}$ ranges from 600 to 840 . We also consider two values for the standard deviation of the measurement errors in the factors $(\sigma), 0.25 \%$ and $1.0 \%$. The second column presents the passing percentages of factors ( $f$ and $g$ ) for the necessary condition hurdle. The third column presents the passing percentages for the sufficient condition, while including the characteristics, $\mathrm{Z}$, in the monthly cross-sectional regressions. ${ }^{17}$

We find that the $\mathrm{f}$ and $\mathrm{g}$ factors all pass the necessary condition at rates in excess of $95 \%$. The average pass rate for the priced $\mathrm{f}$ factors at the sufficient condition (pricing) stage is about $80 \%$ when the number of time periods is low but rises to well above $90 \%$ for the lower value of

\footnotetext{
${ }^{17}$ The second stage pass rates are virtually unaffected by whether the characteristics $\mathrm{Z}$ are included in the regression.
} 
the measurement error as we increase the number of time periods to the upper value. The pass rate for the non-priced $\mathrm{g}$ factors is only about $2-6 \%$ (or lower) across all cases. Finally, the priced characteristics, Z, when included in the sufficient condition regressions are significant more than $95 \%$ of the time. The general finding is that the pass rate of false factors is consistently low across all cases, while the pass rate of true factors is more sensitive to the number of periods than to the number of stocks. Overall, the simulation performs satisfactorily. ${ }^{18}$

In the Internet Appendix, we test out other scenarios, such as how often the protocol accepts false factors formed by portfolios that randomly pick out long-short deciles from within the crosssection of stocks. The pass rate for these portfolios ranges between just four and six percent. In another scenario, we assume the econometrician only observes linear combinations of the true factors, and find that these linear combinations generally have pass rates exceeding $90 \%$.

\section{An Empirical Analysis}

This section presents an application of the suggested protocol using simultaneous monthly return observations over a half century, 1965-2014 inclusive. The sample assets are individual U.S. equities listed on CRSP. We select stocks based on Fama and French (1992).

As candidate factors, we include the five Fama-French (2015) factors; market (Rm-Rf), SMB, HML, profitability (RMW), and investment (CMA), the Carhart (1997) momentum factor $(\mathrm{MOM})$, the risk-free rate (Rf), ${ }^{19}$ a traded liquidity factor (LIQ), and the factors based on shortterm (monthly) and long-term reversals, ST_REV as well as LT_REV, respectively. We also

\footnotetext{
${ }^{18}$ As is the case in much of the literature (e.g., Bai, 2003), we assume in the simulation that the true number of factors is known and matches the number of factor candidates. See Connor and Korajczyk (1993) and Onatski (2009) for econometric approaches to scenarios where the number of factors is unknown.

${ }^{19}$ The risk-free rate proxy (obtained from Federal Reserve Bank of St. Louis) is a three-month Treasury Bill rate. Fluctuations in this proxy can be priced when investors have longer horizons, and these fluctuations are not readily diversifiable.
} 
include the Chen, Roll, and Ross (1986) factors. Specifically, $\Delta$ DP, $\Delta \mathrm{IP}, \Delta \mathrm{TS}$, UNEXPI, and $\Delta \mathrm{EI}$ are the traded versions of the Chen, Roll, and Ross (1986) factors.

We obtain Rm-Rf, SMB, HML, RMW, CMA, MOM, ST_REV, and LT_LEV from Ken French's data library. We construct the traded liquidity factor, and Chen, Roll, and Ross (1986)'s five factors using Cooper and Priestley (2011, henceforth CP)'s methodology. We first obtain the raw CRR factors as follows. The default premium, $\triangle \mathrm{DP}$, is the yield spread between Moody's Baa and Aaa corporate bonds. The growth rate of industrial production, $\Delta \mathrm{IP}$ is $\log \left(\mathrm{IP}_{\mathrm{t}}\right)$ subtracted by $\log \left(\mathrm{IP}_{\mathrm{t}-1}\right)$ where $\mathrm{IP}_{\mathrm{t}}$ is the index of industrial production in month $\mathrm{t} . \Delta \mathrm{TS}$ is the term premium defined as the yield spread between the long-term (10-year) and the one-year Treasury bonds. UNEXPI and $\Delta \mathrm{EI}$ are unexpected inflation and change in expected inflation, respectively. Similar to Chen, Roll and Ross (1986), UNEXPI is derived from the total seasonally adjusted consumer price index (CPI). We collect the inputs for these five factors from the website of Federal Reserve Bank of St. Louis. We obtain Pastor and Stambaugh (2003)'s innovation (INNOV) series from Lubos Pastor's website to construct liquidity-traded portfolio. We do not apply their traded factor (VWF) because it is in fact a zero net investment portfolio formed by longing stocks with high loadings on INNOV and shorting stocks with low loadings. A properly specified factor loading requires a loading of one on the actual factor (the INNOV series) and zero on other factors. This concept is consistent with $\mathrm{CP}$, so we use the $\mathrm{CP}$ method to construct a traded version of the Pastor and Stambaugh's factor using the INNOV series as the input.

In Table 2, we present summary statistics associated with these factor candidates. The liquidity, market, and MOM factors tend to be the most volatile, whereas Rf and the Chen, Roll, and Ross (1986) factors tend to exhibit the least variation (except for industrial production.) The liquidity, momentum, and short-term reversal factors tend to exhibit the highest mean returns. The 
momentum factor has negative skewness, as does the market factor.

To construct mimicking portfolios for six factors (the five CRR-based ones and the liquidity factor), we collect the return of fifty portfolios (the returns of the ten equal-weighted size portfolios, ten equal-weighted book-to-market portfolios, ten value-weighted momentum portfolios, ten equal-weighted investment portfolios and ten equal-weighted operating profitability portfolios) from Ken French's website. We apply CP, who adopt the Lehmann and Modest (1988, Section II) approach as follows. Returns of each of the 50 test assets are regressed on the five CRR factors and INNOV i.e., we perform 50 time-series regressions producing a (50x6) matrix B of slope coefficients against the five CRR factors and INNOV. We generate the variance-covariance matrix of the error terms for these regressions, which is denoted as V. The weight on the mimicking portfolios (W), a 6 x 50 matrix, is computed as $\left(\mathrm{B}^{\prime} \mathrm{V}^{-1} \mathrm{~B}\right)^{-1} \mathrm{~B}^{\prime} \mathrm{V}^{-1} \mathrm{R}$, the returns of 50 portfolios, is a Tx50 matrix where T is a number of months. The return of the CRR mimicking portfolios is WR', a $6 \mathrm{x}$ T matrix, where each row represents the relevant mimicking portfolio return over the sample period. The $\mathrm{CP}$ procedure thus generates mimicking portfolios for each factor, where the beta with respect to a particular factor is unity.

The next step in our protocol is to compute asymptotic principal components that represent the covariance matrix. Because of possible non-stationarity, we split the overall sample into five subsamples with ten years each, while the first spans seven years because one of the potential factors was unavailable for the first three years, 1965-67 inclusive. For each subsample, we extract ten principal components from the return series, using the method of Connor and Korajczyk (CK) (1988). In Table 3 we present the summary statistics for the principal components for each decade of our sample period. The components tend to be skewed, but cases of negative and positive skewness are about equally common. We retain only the first 10 PCs because they account for 
close to $90 \%$ of the cumulative eigenvalues or the total volatility in the covariance matrix, suggesting these 10 PCs capture most of the stock variations. We admit that the number of retained PCs is somewhat arbitrary. If something is omitted, it is omitted for all stocks and should not have impact on the pattern of detected factors. Considering the average across 50 sample years of the cumulative percentage of variance explained within the estimation year, the first principal component explains about $38 \%$ of the variance and five PCs explain over $75 \%$. Thus, this is evidence of multiple factors, not just one. We also find there is some variation in cumulative variance explained within each estimation year by the first 10 PCs from year to year, and the total of variance explained is $90 \%$.

Our protocol then proceeds to calculate canonical correlations. Since we have several factor candidates, there are several pairs of canonical variates, each pair being orthogonal to the others and having a particular intercorrelation. Canonical correlation sorts these pairs from largest to smallest based on their squared correlation, but the correlation itself can be either positive or negative. Panel A of Table 4 reports, in the second and third columns, the canonical correlations for the covariance matrices, and associated t-statistics, covering 1968-1974 monthly. The next few columns provide the correlations and t-statistics for subsequent periods.

As indicated by these results, the first and largest canonical correlation is dominant. Its mean conditional value is close to unity and strongly significant. Across all subperiods, only two correlation falls below .2 in absolute terms. The top five canonical correlations are significant in every subperiod we consider.

Information on significant relations between factor candidates and the principal components is reported in Panel B of Table 4. We use the following procedure to derive the 
significance levels of each factor candidate. First, for each of the ten canonical pairs, ${ }^{20}$ the eigenvector weights for the $10 \mathrm{CK}$ PCs are taken and the weighted average CK PC (which is the canonical variate for the $10 \mathrm{CK}$ PCs that produced the canonical correlation for this particular pair) is constructed. Then, a regression using each CK PC canonical variate as the dependent variable and the actual candidate factors values as independent variables is run over the sample months in each subperiod. The square root of the R-square from this regression (not the adjusted R-square) is the canonical correlation. The coefficients of the regression are equal (after proper normalization) to the eigenvector's weighting elements for the candidate factors. The t-statistics from the regression then give the significance level of each candidate factor. Since there are 10 pairs of canonical variates in each of the five subperiods and a canonical correlation for each one, there is a total of 50 such regressions. The first row presents the mean t-statistic of all canonical correlations. The second row shows the mean t-stat across cases where the canonical correlation itself is statistically significant. The fifth through ninth rows present the number of significant canonical correlations in each decade and the bottom row presents its average.

Since the t-statistics are those of coefficients that represent the square roots of eigenvalues, they are always positive, so a one-tailed cutoff is appropriate. We find that the mean t-statistics for the Fama-French three factors all exceed the one-tailed 2.5\% level cutoff of 1.96. Further, the mean t-statistics for momentum are significant at the 5\% levels for a one-tailed test. The average number of significant t-statistics exceeds two for all factors except CMA, $\Delta \mathrm{IP}, \Delta \mathrm{EI}$, and LIQ.

We adopt the following screening criteria based on Table 4: a candidate is deemed a possible risk factor if in Table 4, Panel B, the average t-statistic of significant canonical correlation in the second row exceeds the one-tailed, $2.5 \%$ cutoff based on Chi-square value, and the average

\footnotetext{
${ }^{20}$ Recall that there are $\min (\mathrm{L}, \mathrm{K})$ possible pairs, and in our application, $\mathrm{L}=10$ and $\mathrm{K}=15$.
} 
number of significant t-stats (last row of Table 4, Panel B) exceeds 2.5 (out of a maximum of five). We focus on the significant canonical correlations, rather than all canonical correlations, because insignificant CCs imply that none of the factors matter, so using them would be over-fitting. This results in nine factors passing the hurdle, including the three original Fama-French (1992) market (Rm-Rf), SMB, HML factors, and one of the two new Fama-French (2015) factors, RMW, followed by MOM, LT_REV, $\triangle \mathrm{DP}, \triangle \mathrm{TS}$, and UNEXPI. These factors pass the screen of being materially related to the covariance matrix of returns across the subperiods we consider. ${ }^{21}$

\section{Are the Factors Priced?}

\subsection{Regression Analysis}

The next step in the protocol is to check whether a factor candidate that passes the necessary conditions is priced in the cross-section of returns. This procedure is as follows: We estimate two versions of factor betas, one uncorrected, and one corrected for EIV. For the non-EIV estimation, OLS multiple regressions are run for each stock on the nine accepted factors using all available observations for that stock. Then, for each calendar month in the sample, January 1965 through December 2014 inclusive, available individual stock returns are multiple-regressed crosssectionally on the nine OLS beta estimates. ${ }^{22}$ The time series averages of the cross-sectional coefficients, termed the "risk premiums," along with associated sampling statistics, are then computed.

\footnotetext{
${ }^{21}$ Note that the investment factor (i.e., CMA, also considered by Hou, Xue, and Zhang, 2015) does not pass the necessary condition, indicating that it is not materially related to the covariance matrix.

${ }^{22}$ A possible check for a priced factor is (i) whether it is related to the covariance matrix and (ii) whether it has a significant mean return. However, because the risk premiums emanate via multiple regression, whereas factor mean returns are in univariate settings (ii) above is not directly comparable to the regression analysis. As an example, if one factor is similar to another factor, it is of interest to determine which of them carries stronger evidence of being priced, and this is best facilitated in a multiple regression.
} 
For the EIV calculations, stocks are sorted into ten groups (deciles) by market capitalization (Size), annually at the end of each June, based on NYSE size decile breakpoints. Then within each Size decile, stocks are sorted further by the OLS betas of the first factor (RmRf) into ten deciles, thus resulting in 100 Size/first factor beta groups. Within each of the 100 groups, the equal weighted average first factor beta of the group is assigned to each stock within that group. This is repeated for each of the eight additional betas whose factors pass the necessary conditions. Hence, for each of the nine factors, the individual stock beta is replaced by the equallyweighted mean beta of the size/beta sorted group to which the stock belongs. Subsequently, over each of the following 12 calendar months, July through June of the next year, all available individual stock returns are multiply-regressed cross-sectionally on the nine EW mean betas assigned to that stock. This is repeated for each June in the sample period, 1965-2014; then the time series average of the cross-sectional coefficients, i.e., the risk premiums, along with associated sampling statistics are computed. There are 594 months in the time series; the first six months are not used because the first sort is done in June 1965 and the last sort in June 2014 has only six available subsequent months.

We also control for stock-specific characteristics corresponding to some of the risk factors (specifically, those that are in fact associated directly with characteristics). The idea is to conduct a horse race between factor betas and the characteristics in the spirit of Daniel and Titman (1997). ${ }^{23}$ The characteristics are RetLag1 (the one month lagged return), Lag2_12 (the two to twelve month lagged return, Lag13_36 (the thirteen to thirty-six months lagged return), Size, Book/Mkt,

\footnotetext{
${ }^{23}$ This horse race is relevant because, as Karolyi (2016) points out, characteristics may be related to factor loadings, and without a proper setting that includes both betas and characteristics, one may misleadingly conclude that a characteristic represents market inefficiency.
} 
ProfRatio (Profitability), AssetGrth (asset growth), and Amihud's (2002) illiquidity measure. These characteristics are defined in detail within the Appendix.

Table 5 reports summary statistics for the (non-EIV-corrected) betas as well as the characteristics. Variables are first averaged cross-sectionally, then in the time series. The default premium's innovation carries the lowest (most negative) mean betas whereas SMB and the market factor have the highest (most positive) mean betas. ${ }^{24}$ Amongst the characteristics, the one-month lagged returns are the most volatile, and prior (2-12) returns also exhibit relatively high variation. SMB also exhibits considerable negative skewness.

Table 6 presents estimated risk premiums for both the non-EIV-corrected and EIVcorrected betas. The first two models present the FM regression for the nine factors that pass our necessary conditions. Rm-Rf, RMW, Momentum and unexpected inflation are the only factors that command a risk premium. [Note that a negative premium of unexpected inflation is expected according to the way it is defined; high unexpected inflation is an adverse event which has a downward impact on stock prices.] Following the EIV correction, the excess market return, RMW, and unexpected inflation are significant.

In Models 3 and 4, we present results from FM regressions with the nine betas on the factors that pass necessary conditions, as well as the characteristics. The results show that Rm-Rf, HML, RMW, LT_Rev, $\triangle \mathrm{DP}$, UNEXPI and $\underline{\text { all }}$ characteristics command significant premiums. After correcting for EIV, the same factors except LT_Rev, UNEXPI, and lag13_36 remain significant. Further, $\Delta \mathrm{TS}$ becomes significant in this specification. Surprisingly, the HML risk premium is negative and significant, presumably because the beta estimates of HML (and CMA)

\footnotetext{
${ }^{24}$ Note that the mean multivariate beta for UNEXPI is positive, which is counterintuitive because one would expect inflation shocks to negatively affect stock returns. In a univariate setting, the average UNEXPI beta is indeed negative; the positive sign is the result of the interaction of this factor with others.
} 
are contaminated by multicollinearity. Indeed, we find that the correlation between HML and CMA is .71. It is noteworthy that in the EIV-corrected regression the insignificance result for lag13_36 is consistent with that of long-term reversals.

The Amihud measure causes a loss in sample size of more than $50 \%$. Hence we do not report results that include this measure. However, in unreported analyses, we do run regressions with this measure (along with all of the other betas and characteristics), and find that the results remain qualitatively unchanged. These results appear in the Internet Appendix.

We note that the characteristics in general are far more significant than the betas. While we cannot dismiss the possibility that characteristics instrument for unidentified risk exposures (Berk, 2000; Zhang, 2005), the results are consistent with market inefficiency.

We note that there is no major difference between correcting for EIV and not doing so. The results for the simplest cross-sectional regression risk premium estimates match the estimates using double sorts on all candidate factors (double sorts on size and beta and then portfolio betas replacing individual stock betas.) The Fama-French (1992) method introduces its own EIV problem; using portfolio betas in place of individual stock betas. This is an EIV since the true but unknown individual stock beta is not used. ${ }^{25}$

\subsection{Hedge Portfolio Returns}

While in Section 5, we describe the desirability of using individual securities as test assets, in this subsection we perform a robustness check with the standard methodology of forming hedge

\footnotetext{
${ }^{25}$ An instrumental variables (IV) approach represents another approach for resolving the EIV problem. It is potentially implementable by choosing odd month betas for instruments and even month betas for regressors, or vice versa (Jegadeesh et al., 2017). We did not employ it here because we were wary of the "weak instrument" issue in IV analysis, which might impact odd or even beta estimates used as instruments. To avoid any potential problem in this regard, we resorted to other methods.
} 
portfolios. These are formed by going long (short) in the portfolios with the highest (lowest) beta in deciles. ${ }^{26}$ Table 7 Panel A presents the hedge portfolios that are long the top decile and short the bottom decile after sorting by individual stock (EIV-corrected) betas on each factor with replacement. For example, the individual stock betas on $\mathrm{Rm}-\mathrm{Rf}$ are sorted and a portfolio is formed from the stocks with the largest $10 \%$, equal weighted, and then another portfolio is formed from the stocks with the smallest $10 \%$. The second portfolio's return is subtracted from the first one. Then, for SMB, the same procedure is repeated. Individual SMB betas are sorted and then the hedge portfolio's return comes from the largest less the smallest decile. This is repeated for each candidate factor. The results, presented in Table 7, Panel A indicate that hedge portfolios for the market and RMW factors remain significant, whereas all of the Chen, Roll, and Ross (1986) factors, namely the innovations to the default premium, term spread, and inflation, also are significant. The sign of the hedged portfolio returns for unexpected inflation is negative. This is consistent with the sign of its risk premium in Table 6 .

The final step in our protocol checks for whether the Sharpe ratios generated by the factors are within reasonable magnitudes. The traded versions of the factors are zero net investment portfolios. We thus combine each of them with a representative long-only portfolio in order to check if the Sharpe ratio is below a reasonable bound, relative to a representative long-only equity portfolio. Accordingly, in Panel B, we present the mean, standard deviation, and Sharpe ratios for a portfolio that combines the market (i.e., the value-weighted CRSP index) with the zero net investment long-short portfolio from Panel A and tests whether the resulting Sharpe ratio is statistically greater than the bound of 0.6 recommended by MacKinlay (1995, p.13). None of the

\footnotetext{
${ }^{26}$ Kozak, Nagel, and Santosh (2018) argue that a return spread from sorted betas is not necessarily evidence that the associated factor is actually "priced" due to risk because behavioral biases can affect factor loadings; in turn, this implies that systematic investor mistakes can be reflected in factor pricing.
} 
Sharpe ratios exceed this threshold; indeed, eight of nine are below the threshold. Thus, the priced factors command Sharpe ratios of a magnitude consistent with risk-based pricing.

Panels C and D repeat the analyses of Panels A and B, except that the long-short portfolios are based on the top (bottom) $30 \%$ of stocks with the highest and lowest betas, instead of the highest and lowest deciles of betas. The results are similar. In Panel D, almost all of the Sharpe ratios are lower than the MacKinlay (1995) threshold.

Table 8 presents hedge portfolio results similar to those in Table 7, but using characteristics rather than factor betas as the sorting criteria. Panel A demonstrates that RetLag1 (monthly reversals), Lag2_12 (momentum), Book/Mkt, ProfRato, and AsstGrth provide statistically significant average returns. The negative sign for RetLag1 and AssetGrth is consistent with their negative risk premium in Table 8. Panel B tests whether the Sharpe ratio from combining the market and the zero net investment portfolio from Panel A is greater than the MacKinlay bound of 0.6. The results show that RetLag1, Lag2_12, Book/Mkt and ProfRato provide SRs that are statistically higher than the bound. That is, the strategies associated with these characteristics, although linked with premiums, provide abnormally high Sharpe ratios. When we construct hedge portfolios using the top and bottom $30 \%$ of stocks in Panel C, the results are similar to those in Panel A. The Sharpe ratios in Panel D also have largely similar patterns as those in Panel B except that only Lag2_12 and Book/Mkt yield SR greater than 0.6. ${ }^{27}$

Table 9 presents the cross-correlations obtained using the hedge portfolios formed in Panel A of Tables 7 and 8, across the factors and the characteristics. $86 \%$ of the correlations are below

\footnotetext{
${ }^{27}$ Note that the sample observations used are different for different runs. The non-EIV sample sizes are different from the doublesorted EIV sample sizes and the double-sort approach cannot be the same because it uses portfolio betas to replace the individual stock betas. This means that some observations are available after the double size sorts even when the individual betas are not available; e.g., because we require 24 observations to compute them. The hedge portfolios are formed by sorting on betas or characteristics one variable at a time. Obviously, the beta sorted hedge portfolios and characteristics sorted hedge portfolios have different stocks and, equally obviously, the particular stocks within the $10 \%$ and $30 \%$ portfolios are different for each beta and characteristic.
} 
0.5 in absolute magnitude. The hedge portfolio for the profitability characteristic is negatively correlated with SMB, whereas HML, RMW, and MOM, not surprisingly, are positively correlated with their characteristic-based counterparts. The hedge portfolios corresponding to some of the CRR factors are also positively cross-correlated with some characteristic-based portfolios, but there is no ready explanation for these results, so we leave a full explanation for future research.

Overall, when subjected to our protocol, across both the regression and hedge portfolio method, a market factor, a profitability factor, and factors based on credit spreads, term spread, and unexpected inflation are related to the covariance matrix, command statistically significant risk premiums in all specifications, and yield reasonable Sharpe ratios. Almost all characteristics are associated with statistically significant premiums, but only momentum and the book/market anomaly yield Sharpe ratios that exceed a reasonable bound to be considered an abnormal profit opportunity.

\section{Summary and Conclusions}

Our goal in this paper is to suggest a protocol for categorizing factors that potentially are the drivers of asset returns and for determining whether they are associated with risk premiums. We are striving for a procedure that will be acceptable to scholars and practitioners; a standard for future factor identification. The protocol we present here is just an outline and it will undoubtedly be modified by others to render it more acceptable. Ours is just a first attempt.

Our suggested protocol has two stages. The first stage provides a sequence of steps that represent necessary conditions for factor candidates to be valid. A candidate that does not satisfy these conditions is not a risk factor, but this does not imply that rejected candidate is uninteresting, particularly to investors. Indeed, if such a rejected candidate is related to average returns on any 
set of assets, there is a potential profit opportunity. In principle, a diversified portfolio could be constructed to produce significant return with minimal risk. The second suggested stage entails testing whether factor candidates that satisfy the necessary conditions are pervasive and consequently have associated risk premiums or instead are unpriced in the cross-section.

One very important application of our protocol would be to study the relative importance of industry, country, and global factors. Intuitively, some factors might be pervasive globally but there is some doubt because many or perhaps most countries do not share fully integrated macroeconomic systems. This leaves room for country factors and, indeed, most previous studies of factors have been exclusively domestic. Finally, at an even lower level of aggregation, industry factors clearly have the ability to explain some individual firm covariances; but do they all carry no risk premiums or, instead, are at least some of them sufficiently pervasive to be genuine risk factors at either the country or global level?

Industry factors have been studied for a long time, from King (1966) through Moskowitz (2003). It seems to us that a very useful exercise would be to study industry factors globally. Following our suggested protocol, we would only need to assemble some international real asset returns, extract a time series of eigenvectors from their time-varying covariances, and check whether industry factors satisfy the necessary and sufficient conditions of Sections 4 and 5 above. 


\section{References}

Amihud, Y. 2002. Illiquidity and stock returns: Cross-section and time-series effects. Journal of Financial Markets 5:31-56.

Anderson, T. W. 1984. An introduction to multivariate statistical analysis. $2^{\text {nd }}$ ed. New York: Wiley.

Arnott, R., J. Hsu, and P. Moore. 2005. Fundamental indexation. Financial Analysts Journal 61: 83-99.

Bai, J. 2003. Inferential theory for factor models of large dimensions. Econometrica 71:135-171.

Barillas, F., and J. Shanken. 2018. Comparing asset pricing models. Journal of Finance 73:715754.

Belloni, A., V. Chernozhukov, and C. Hansen. 2014. Inference on treatment effects after selection among high-dimensional controls. Review of Economic Studies 81:608-650.

Berk, J. B. 2000. Sorting out sorts. Journal of Finance 55:407-427.

Brennan, M. C., T. Chordia, and A. Subrahmanyam. 1998. Alternative factor specifications, security characteristics and the cross section of expected stock returns. Journal of Financial Economics 49:345-373.

Brown, S. 1989. The number of factors in security returns. Journal of Finance 44:1247-1262.

Campbell, J. Y., and J. H. Cochrane. 2000. Explaining the poor performance of consumption-based asset pricing models. Journal of Finance 55:2863-2878.

Campbell, J. Y., S. Giglio, C. Polk, and R. Turley. 2017. An intertemporal CAPM with stochastic volatility. Forthcoming Journal of Financial Economics.

Campbell, J. Y., M. Lettau, B. Malkiel, and Y. Xu. 2001. Have individual stocks become more volatile? An empirical exploration of idiosyncratic risk. Journal of Finance 56:1-43.

Carhart, M. M. 1997. On persistence in mutual fund performance. Journal of Finance 52:57-82.

Charoenrook, A., and J. Conrad. 2008. Identifying Risk-Based Factors. Working paper, Vanderbilt University.

Chen, N., R. Roll, and S. A. Ross. 1986. Economic forces and the stock market. Journal of Business 59:383-403.

Cochrane, J. H. 2001. Asset Pricing. Princeton: Princeton University Press. 
Connor, G., and R. A. Korajczyk. 1988. Risk and return in an equilibrium APT: Application of a new test methodology. Journal of Financial Economics 21:255-289.

Connor, G., and R. A. Korajczyk. 1993. A test for the number of factors in an approximate factor model. Journal of Finance 48:1263-1291.

Cooper, I., and R. Priestley. 2011. Real investment and risk dynamics. Journal of Financial Economics 101:192-205.

Cotter, J., and R. Roll. 2015. A comparative anatomy of REITs and residential real estate indexes: Returns, risks and distributional characteristics. Real Estate Economics 43:209-240.

Daniel, K. and S. Titman. 1997. Evidence of the characteristics of cross sectional variation in Stock returns. Journal of Finance 52:1-33.

Engelberg, J., R. D. McLean, and J. Pontiff. 2017. Anomalies and news. Forthcoming Journal of Finance.

Fama, E., and K. French. 1992. The cross-section of expected stock returns. Journal of Finance 47:427-466.

Fama E., and K. French. 1993. Common risk factors in the returns on stocks and bonds. Journal of Financial Economics 33:3-56.

Fama, E., and K. French. 2008. Dissecting anomalies. Journal of Finance 63:1653-1678.

Fama, E., and K. French. 2015. A Five-factor asset pricing model. Journal of Financial Economics 116:1-22.

Fama, E., and K. French. 2017. Choosing Factors. Forthcoming Journal of Financial Economics.

Fama, E., and J. D. MacBeth. 1973. Risk, return, and equilibrium: Empirical tests. Journal of Political Economy 81:607-636.

Feng, G., S. Giglio, and D. Xiu, 2017. Taming the Factor Zoo. Working paper, University of Chicago

Green, J., J. Hand, and X. F. Zhang. 2013. The supraview of return predictive signals. Review of Accounting Studies 18:692-730.

Green, J., J. Hand, and X. F. Zhang. 2017. The characteristics that provide independent information about average U.S. monthly stock returns. Review of Financial Studies 30:4389-4436.

Harvey, C.R., and Y. Liu. 2016. Lucky factors. Working paper, Duke University. 
Harvey, C. R., and Y. Liu, and H. Zhu. 2016. ... and the cross-section of expected returns. Review of Financial Studies 29:5-68.

Hou, K., C. Xue, and L.Zhang. 2015. Digesting anomalies: An investment approach. Review of Financial Studies 28:650-705.

Jegadeesh, N., J. Noh, K. Pukthuanthong, R. Roll, and J. Wang. 2017. Empirical tests of asset pricing models with individual assets: Resolving the errors-in-variables bias in risk premium estimation. Forthcoming Journal of Financial Economics.

Johnson, R. A., and D. W. Wichern. 2007. Applied Multivariate Statistical Analysis. $6^{\text {th }}$ ed. New York: Prentice-Hall.

Karolyi, G. A. 2016. The cross-section of expected returns: Where we stand today. Review of Financial Studies 29:2-4.

King, B. F. 1966. Market and industry factors in stock price behavior. Journal of Business 39:139190.

Kozak, S., S. Nagel, and S. Santosh. 2018. Interpreting factor models. Journal of Finance 13:11831223.

Lehmann, B., and D. Modest. 1988. The empirical foundations of the arbitrage pricing theory. Journal of Financial Economics 21:213-254.

Lewellen, J. 2015. The cross-section of expected stock returns. Critical Finance Review 4:1-44.

Lewellen, J., and S. Nagel. 2006. The conditional CAPM does not explain asset-pricing anomalies. Journal of Financial Economics 82:289-314.

Lewellen, J., S. Nagel, and J. Shanken, 2010. A skeptical appraisal of asset pricing tests. Journal of Financial Economics 96:175-194.

Linnainmaa, J., and M. R. Roberts. 2017. The history of the cross section of stock returns. forthcoming Review of Financial Studies.

Lo, A., and A. C. MacKinlay. 1990. When are contrarian profits due to stock market overreaction? Review of Financial Studies 3:175-205.

MacKinlay, A. C. 1995. Multifactor models do not explain deviations from the CAPM. Journal of Financial Economics 38:3-28.

McLean, R. D., and J. Pontiff. 2016. Does academic research destroy stock return predictability? Journal of Finance 71:5-31.

Moskowitz, T. J. 2003 An analysis of covariance risk and pricing anomalies. The Review of Financial Studies 16:417-457. 
Onatski, A. 2000. Testing hypotheses about the number of factors in large factor models. Econometrica 77:1447-1470.

Pastor, L., and R. F. Stambaugh. 2003. Liquidity risk and expected stock returns. Journal of Political Economy 111:642-685.

Petersen, M. A. 2009. Estimating standard errors in finance panel data sets: Comparing approaches. Review of Financial Studies 22:435-480.

Roll, R. 1977. A Critique of the asset pricing theory's test. Journal of Financial Economics 4:129176.

Roll, R., and S. A. Ross. 1980. An empirical investigation of the arbitrage pricing theory. Journal of Finance 35:1073-1103.

Ross, S. 1976. The arbitrage theory of capital asset pricing. Journal of Economic Theory 13:341360.

Singleton, K. J. 2006. Empirical dynamic asset pricing: Model specification and econometric Assessment. Princeton University Press.

Subrahmanyam, A. 2010. The cross-section of expected stock returns: What have we learnt from the past twenty-five years of research? European Financial Management 16:27-42.

Zhang, L. 2005. The value premium. Journal of Finance 60:67-103. 


\section{Appendix}

\section{Variable Definition}

\begin{tabular}{|c|c|}
\hline Variables & Description \\
\hline $\mathrm{Rm}-\mathrm{Rf}$ & Market excess return factor from Ken French's library \\
\hline SMB & Small minus big factor from Ken French's library \\
\hline HML & High minus low B/M factor from Ken French's library \\
\hline RMW & Robust minus weak operating profitability from Ken French's library \\
\hline CMA & Conservative minus aggressive investment from Ken French's library \\
\hline MOM & Momentum factor from Ken French's library \\
\hline Rf & 3-month Treasury Bill rate from Federal Reserve Bank of St. Louis \\
\hline LIQ & $\begin{array}{l}\text { Traded factor of liquidity constructed from Pastor and Stambaugh (2003)'s innovation series, collected from Lubos Pastor's } \\
\text { website }\end{array}$ \\
\hline ST_Rev & Short-term reversal factor from Ken French's library \\
\hline LT_Rev & Long-term reversal factor from Ken French's library \\
\hline$\Delta \mathrm{DP}$ & $\begin{array}{l}\text { Traded factor of default risk premium where default risk premium is the yield spread between Moody's Baa and Aaa } \\
\text { corporate bonds from Federal Reserve Bank of St. Louis }\end{array}$ \\
\hline$\Delta \mathrm{IP}$ & $\begin{array}{l}\text { Traded factor of growth rate of industrial production, where industrial production is from the Federal Reserve Bank of St. } \\
\text { Louis }\end{array}$ \\
\hline$\Delta \mathrm{TS}$ & $\begin{array}{l}\text { Traded factor of term premium where term premium is the yield spread between the ten-year and the one-year Treasury } \\
\text { bonds from Federal Reserve Bank of St. Louis }\end{array}$ \\
\hline UNEXPI & $\begin{array}{l}\text { Traded factor of unexpected inflation where unexpected inflation at time } t \text { is the difference between inflation at time } t \text { and } \\
\text { expected inflation at time } t-1 \text {. Both are available from the Federal Reserve Bank of St. Louis }\end{array}$ \\
\hline$\Delta \mathrm{EI}$ & Traded factor of change in expected inflation, where expected inflation is from the Federal Reserve Bank of St. Louis \\
\hline RetLag1 & Return in prior month from CRSP $(\%)$ \\
\hline Lag2_12 & Return in prior 2nd to $12^{\text {th }}$ month from CRSP (in \% per month) \\
\hline Lag13_36 & Return in prior 13th to $36^{\text {th }}$ month from CRSP (in \% per month) \\
\hline SizeLag1 & $\begin{array}{l}\text { Natural log of size (market cap) lagged one-month relative to the return. For instance, if the return is for February } 1965 \text {, } \\
\text { SizeLag1 is the log market cap at the end of January } 1965 \text {. Price and number of shares outstanding are from CRSP. }\end{array}$ \\
\hline Book/Mkt & $\begin{array}{l}\text { Book-to-market equity, the natural log of the ratio of the book value of equity to the market value of equity. Book equity } \\
\text { is total assets (Compustat data item } 6 \text { ) for year t-1, minus liabilities (181), plus balance sheet deferred taxes and investment } \\
\text { tax credit (35) if available, minus preferred stock liquidating value (10) if available, or redemption value (56) if available, } \\
\text { or carrying value (130). Market equity is price times shares outstanding at the end of December of t-1, from CRSP. }\end{array}$ \\
\hline
\end{tabular}




\begin{tabular}{|c|l|}
\hline ProfRato & $\begin{array}{l}\text { Profit ratio for June of year } \mathrm{t} \text { is annual revenues minus cost of goods sold, interest expense, and selling, general, and } \\
\text { administrative expenses divided by book equity for the last fiscal year end in } \mathrm{t}-1 .\end{array}$ \\
\hline AsstGrth & $\begin{array}{l}\text { Annual firm asset growth rate is calculated using the year-on-year percentage change in total assets (Compustat data item } \\
\text { 6). The firm asset growth rate for year t is estimated as the percentage change in data item } 6 \text { from fiscal year ending in } \\
\text { calendar year t-2 to fiscal year ending in calendar year t-1, }\end{array}$ \\
\hline $\begin{array}{c}\text { Amihud's } \\
\text { illiquidity } \\
\text { ratio }\end{array}$ & $\begin{array}{l}\text { The annual illiquidity ratio of stock i in year t measured as the average ratio of the daily absolute return to the (dollar) } \\
\text { trading volume on that day divided by number of days for which data are available for stock i in year t. }\end{array}$ \\
\hline
\end{tabular}


Table 1

\section{Simulation Results}

This table presents the percentage pass rates for the necessary condition (Columns (1)) and the sufficient condition (Columns $(2))$ from 5,000 simulations. f. 1 to $\mathrm{f} .5$ are factors that are associated with risk premiums. Factors g.1, g.2, and g. 3 do not command risk premiums. $Z_{\mathrm{i}}(\mathrm{i}=1, \ldots .5)$ represents five characteristics. f.1 has a mean and standard deviation equal to those of the excess return on the value-weighted CRSP index. The factors f.2 to f.5 are generated independently from a distribution with the actual mean and standard deviation of HML. Factors g are independent and have mean zero, with standard deviations equal to that of HML. The five characteristics represent firm-specific information that varies across time, and they are generated at each time period from the uniform distribution $[-0.1 \%, 0.1 \%]$. The standard deviation for the error term is computed to ensure that, for a "typical" stock whose betas equal their cross-sectional means, the $R^{2}$ from a regression of individual stock returns on the factors equals $25 \%$. The simulation is based on 600,720 , and 840 periods of time and standard deviation of measurement errors $\eta_{i, t}$ and $\iota_{i, t}$ of $\tilde{f}_{i, t}$ and $\tilde{g}_{i, t}$, respectively (see Section 6) of 1\% and $0.25 \%$ for 2,000 stocks (Panel A), 3000 stocks (Panel B), and 6000 stocks (Panel C).

Panel A: Number of stocks $=2,000$

\begin{tabular}{|c|c|c|c|c|c|c|c|c|c|c|c|c|}
\hline \multirow[t]{3}{*}{$\begin{array}{c}\text { Factor } \\
\text { candidates }\end{array}$} & Perioc & ime $=$ & \multicolumn{2}{|c|}{$\begin{array}{c}\text { Periods of time }= \\
720\end{array}$} & \multicolumn{2}{|c|}{$\begin{array}{c}\text { Periods of time }= \\
840\end{array}$} & \multicolumn{2}{|c|}{$\begin{array}{c}\text { Periods of time }= \\
600\end{array}$} & \multicolumn{2}{|c|}{$\begin{array}{l}\text { Periods of } \\
\text { time }=720\end{array}$} & \multicolumn{2}{|c|}{$\begin{array}{c}\text { Periods of time }= \\
840\end{array}$} \\
\hline & \multicolumn{6}{|c|}{ Standard deviation of measurement errors $=1.00 \%$} & \multicolumn{6}{|c|}{ Standard deviation of measurement errors $=0.25 \%$} \\
\hline & $(1)$ & $(2)$ & $(1)$ & $(2)$ & $(1)$ & $(2)$ & $(1)$ & $(2)$ & $(1)$ & $(2)$ & $(1)$ & $(2)$ \\
\hline f.1 & 100 & 73 & 100 & 82 & 100 & 86 & 100 & 75 & 100 & 80 & 100 & 90 \\
\hline f. 2 & 100 & 83 & 100 & 88 & 100 & 90 & 100 & 81 & 100 & 89 & 100 & 92 \\
\hline f. 3 & 100 & 81 & 100 & 87 & 100 & 91 & 100 & 83 & 100 & 87 & 100 & 91 \\
\hline f. 4 & 100 & 82 & 100 & 89 & 100 & 94 & 100 & 82 & 100 & 89 & 100 & 93 \\
\hline f. 5 & 100 & 83 & 100 & 89 & 100 & 92 & 100 & 84 & 100 & 88 & 100 & 94 \\
\hline g. 1 & 100 & 2.6 & 100 & 6.2 & 100 & 4.2 & 100 & 4.0 & 100 & 4.8 & 100 & 3.2 \\
\hline g.2 & 100 & 3.6 & 100 & 3.6 & 100 & 4.8 & 100 & 4.8 & 100 & 5.4 & 100 & 5.8 \\
\hline g. 3 & 100 & 3.0 & 100 & 4.8 & 100 & 5.2 & 100 & 6.2 & 100 & 3.8 & 100 & 4.8 \\
\hline$Z_{1}$ & - & 98 & - & 100 & - & 99 & - & 99 & - & 99 & - & 100 \\
\hline$Z_{2}$ & - & 98 & - & 99 & - & 100 & - & 97 & - & 100 & - & 100 \\
\hline$Z_{3}$ & - & 99 & - & 100 & - & 99 & - & 98 & - & 100 & - & 100 \\
\hline$Z_{4}$ & - & 99 & - & 99 & - & 100 & - & 98 & - & 99 & - & 99 \\
\hline$Z_{5}$ & - & 98 & - & 99 & - & 100 & - & 99 & - & 99 & - & 100 \\
\hline
\end{tabular}


Panel B: Number of stocks $=3,000$

\begin{tabular}{|c|c|c|c|c|c|c|c|c|c|c|c|c|}
\hline & \multicolumn{2}{|c|}{$\begin{array}{c}\text { Periods of time }= \\
600\end{array}$} & \multicolumn{2}{|c|}{$\begin{array}{c}\text { Periods of time }= \\
720\end{array}$} & \multicolumn{2}{|c|}{$\begin{array}{c}\text { Periods of time }= \\
840\end{array}$} & \multicolumn{2}{|c|}{$\begin{array}{c}\text { Periods of time }= \\
600\end{array}$} & \multicolumn{2}{|c|}{$\begin{array}{c}\text { Periods of time }= \\
720\end{array}$} & \multicolumn{2}{|c|}{$\begin{array}{c}\text { Periods of time }= \\
840\end{array}$} \\
\hline & \multicolumn{6}{|c|}{ Standard deviation of measurement errors $=1.00 \%$} & \multicolumn{6}{|c|}{ Standard deviation of measurement errors $=0.25 \%$} \\
\hline & $(1)$ & $(2)$ & $(1)$ & $(2)$ & $(1)$ & $(2)$ & $(1)$ & $(2)$ & $(1)$ & $(2)$ & $(1)$ & $(2)$ \\
\hline f.1 & 100 & 76 & 100 & 79 & 100 & 89 & 100 & 75 & 100 & 81 & 100 & 90 \\
\hline f. 2 & 100 & 83 & 100 & 89 & 100 & 93 & 100 & 81 & 100 & 90 & 100 & 93 \\
\hline f. 3 & 100 & 82 & 100 & 88 & 100 & 94 & 100 & 82 & 100 & 89 & 100 & 93 \\
\hline f. 4 & 100 & 82 & 100 & 88 & 100 & 94 & 100 & 79 & 100 & 88 & 100 & 92 \\
\hline f. 5 & 100 & 84 & 100 & 89 & 100 & 92 & 100 & 81 & 100 & 87 & 100 & 91 \\
\hline g. 1 & 100 & 2.0 & 100 & 4.2 & 100 & 6.6 & 100 & 4.0 & 100 & 3.6 & 100 & 5.4 \\
\hline g. 2 & 100 & 3.2 & 100 & 3.8 & 100 & 5.0 & 100 & 5.2 & 100 & 6.2 & 100 & 4.4 \\
\hline g. 3 & 100 & 3.2 & 100 & 4.6 & 100 & 5.2 & 100 & 4.6 & 100 & 6.4 & 100 & 6.2 \\
\hline$Z_{1}$ & - & 100 & - & 100 & - & 100 & - & 100 & - & 100 & - & 100 \\
\hline$Z_{2}$ & - & 100 & - & 100 & - & 100 & - & 100 & - & 100 & - & 100 \\
\hline$Z_{3}$ & - & 100 & - & 100 & - & 100 & - & 100 & - & 100 & - & 100 \\
\hline$Z_{4}$ & - & 100 & - & 100 & - & 100 & - & 100 & - & 100 & - & 99 \\
\hline$Z_{5}$ & - & 100 & - & 100 & - & 100 & - & 100 & - & 100 & - & 100 \\
\hline
\end{tabular}

Panel C: Number of stocks $=6,000$

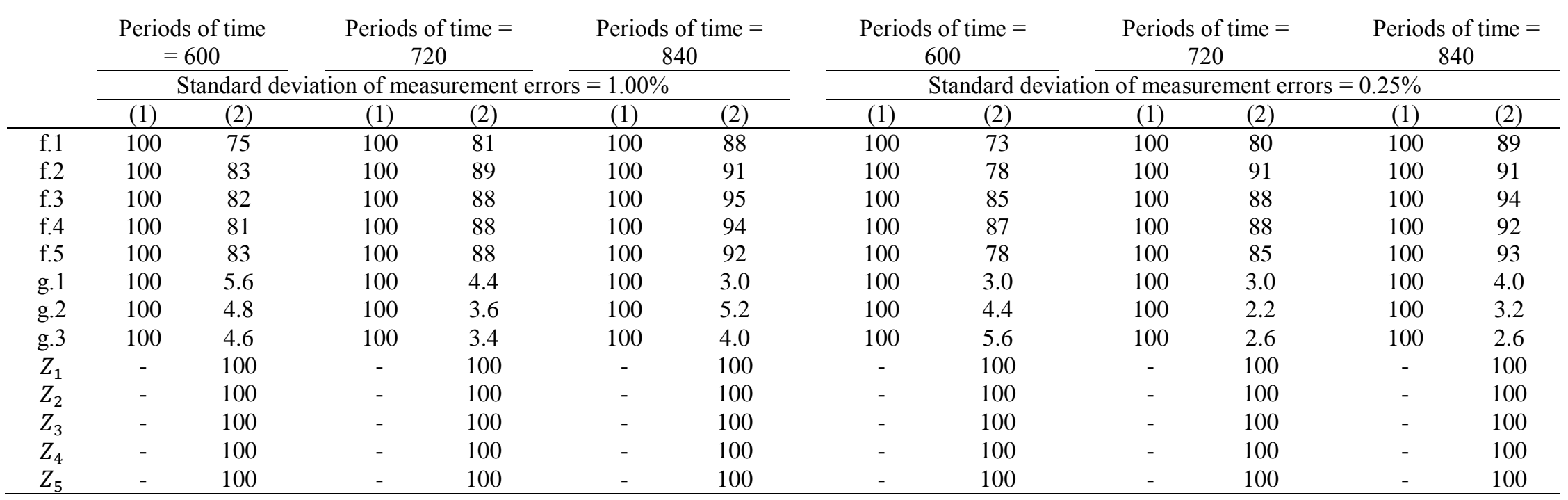


Table 2

\section{Summary Statistics for the Candidate Factors}

Here are summary statistics for candidate factor realizations in \% per month. The sample period spans 600 months from January 1965 through December 2014. See the Appendix for variable definitions.

\begin{tabular}{lccccccc} 
& Mean & Median & Sigma & Skewness & Kurtosis & Maximum & Minimum \\
\hline Rm-Rf & 0.492 & 0.840 & 4.513 & -0.524 & 1.823 & 16.100 \\
SMB & 0.289 & 0.115 & 3.116 & 0.380 & 3.494 & 19.180 & -15.360 \\
HML & 0.354 & 0.335 & 2.897 & 0.000 & 2.583 & 13.910 & -13.110 \\
RMW & 0.253 & 0.165 & 2.163 & -0.403 & 11.22 & 12.190 & -17.570 \\
CMA & 0.324 & 0.195 & 2.038 & 0.269 & 1.585 & 9.510 & -6.810 \\
Rf & 0.411 & 0.410 & 0.261 & 0.554 & 0.815 & 1.350 & 0.000 \\
MOM & 0.690 & 0.775 & 4.283 & -1.401 & 10.77 & 18.380 & -34.580 \\
ST_Rev & 0.497 & 0.330 & 3.180 & 0.367 & 5.459 & 16.200 & -14.580 \\
LT_Rev & 0.301 & 0.185 & 2.534 & 0.629 & 2.628 & 14.490 & -7.790 \\
$\Delta$ DP & -0.061 & -0.066 & 0.267 & -0.017 & 1.913 & 1.050 & -1.361 \\
$\Delta$ IP & 0.375 & 0.419 & 2.893 & -0.316 & 1.496 & 12.670 & -12.810 \\
$\Delta$ TS & -0.099 & -0.098 & 0.876 & 0.143 & 0.590 & 3.093 & -3.190 \\
UNEXPI & -0.203 & -0.203 & 0.783 & -0.025 & 0.487 & 3.011 & -3.121 \\
$\Delta$ EI & 0.012 & 0.011 & 0.118 & 0.062 & 1.401 & 0.575 \\
LIQ & 1.610 & 2.344 & 16.90 & -0.096 & 2.807 & 91.310 \\
\hline
\end{tabular}


Table 3

\section{Summary Statistics for Principal Components}

Here are summary statistics over 600 months for principal components (PCs) extracted using the Connor and Korajczyk (CK) (1988) method. The entire data period spans January 1965 through December 2014, 50 years of monthly observations. For each decade within the fifty years, the CK method is applied to all available stocks with full records, and ten principal components are extracted. The number of stocks included is 1,259 in 1965-1974, 2,331 in 1975-1984, 2,660 in 1985-1994, 3,145 in 1995-2004, and 3,349 in 2005-2014. Each PC has a mean of exactly zero and is normalized to have the same standard deviation. The standard deviation of each PC is 0.092 .

\begin{tabular}{|c|c|c|c|c|c|c|c|c|c|c|c|c|c|c|c|c|c|c|c|c|}
\hline & \multicolumn{4}{|c|}{$1965-1974$} & \multicolumn{4}{|c|}{$1975-1984$} & \multicolumn{4}{|c|}{ 1985-1994 } & \multicolumn{4}{|c|}{$1995-2004$} & \multicolumn{4}{|c|}{$2005-2014$} \\
\hline & Median & $\begin{array}{c}\text { Skew } \\
\text { ness }\end{array}$ & Max & Min & Median & $\begin{array}{c}\text { Skew } \\
\text { ness }\end{array}$ & Max & Min & Median & $\begin{array}{c}\text { Skew } \\
\text { ness }\end{array}$ & Max & Min & Median & $\begin{array}{c}\text { Skew } \\
\text { ness }\end{array}$ & Max & Min & Median & $\begin{array}{c}\text { Skew } \\
\text { ness }\end{array}$ & Max & Min \\
\hline PC1 & 0.005 & -0.053 & 0.258 & -0.245 & 0.038 & -1.200 & 0.115 & -0.206 & -0.002 & -3.851 & 0.360 & -0.733 & 0.002 & 0.477 & 0.387 & -0.263 & -0.004 & 0.087 & 0.358 & -0.389 \\
\hline $\mathrm{PC} 2$ & -0.005 & 0.327 & 0.359 & -0.311 & 0.004 & -0.784 & 0.313 & -0.460 & -0.009 & 3.678 & 0.657 & -0.171 & 0.012 & -3.941 & 0.208 & -0.696 & -0.006 & -0.011 & 0.454 & -0.433 \\
\hline PC3 & 0.019 & -2.675 & 0.155 & -0.602 & 0.001 & 2.949 & 0.666 & -0.246 & -0.009 & 10.063 & 0.968 & -0.051 & -0.003 & 3.068 & 0.627 & -0.306 & 0.004 & -1.214 & 0.548 & -0.655 \\
\hline PC4 & -0.001 & 0.900 & 0.455 & -0.309 & -0.004 & -0.321 & 0.334 & -0.442 & -0.014 & 3.945 & 0.506 & -0.168 & -0.008 & 3.510 & 0.689 & -0.281 & 0.008 & -0.849 & 0.551 & -0.593 \\
\hline PC5 & 0.002 & 1.157 & 0.533 & -0.308 & -0.006 & 0.707 & 0.359 & -0.222 & 0.004 & -0.244 & 0.304 & -0.284 & 0.003 & -3.006 & 0.455 & -0.709 & -0.005 & 3.182 & 0.619 & -0.355 \\
\hline PC6 & -0.006 & 0.550 & 0.286 & -0.226 & 0.002 & -1.608 & 0.328 & -0.579 & 0.001 & -2.508 & 0.236 & -0.589 & 0.005 & 0.507 & 0.450 & -0.268 & -0.006 & 1.354 & 0.443 & -0.389 \\
\hline PC7 & 0.009 & -0.736 & 0.271 & -0.343 & -0.011 & 0.709 & 0.381 & -0.247 & 0.002 & -0.856 & 0.434 & -0.503 & -0.010 & 1.303 & 0.513 & -0.324 & -0.001 & 0.391 & 0.523 & -0.503 \\
\hline PC8 & -0.008 & 0.970 & 0.396 & -0.260 & -0.004 & 2.366 & 0.599 & -0.271 & 0.001 & -0.083 & 0.395 & -0.344 & -0.005 & 4.901 & 0.780 & -0.268 & -0.002 & 0.529 & 0.387 & -0.327 \\
\hline PC9 & 0.005 & -0.320 & 0.295 & -0.326 & 0.001 & 1.807 & 0.630 & -0.366 & 0.006 & -0.274 & 0.364 & -0.384 & 0.002 & -0.932 & 0.305 & -0.428 & -0.004 & -1.519 & 0.466 & -0.620 \\
\hline PC10 & 0.007 & -0.177 & 0.271 & -0.247 & -0.001 & -0.102 & 0.297 & -0.307 & 0.001 & -0.021 & 0.412 & -0.390 & 0.005 & -0.825 & 0.430 & -0.509 & 0.006 & -0.943 & 0.354 & -0.386 \\
\hline
\end{tabular}


Table 4

\section{Canonical Correlations with Asymptotic PCs and Significance Levels of Factor Candidates}

This table reports canonical correlations between Factor Candidates and Principal Components. The factor candidates include the five Fama-French (2015) factors, Rm-Rf, SMB, HML, RMW, and CMA along with RF, MOM, ST_REV, LT_REV, LIQ, $\Delta D P, \Delta I P, \Delta T S$, UNEXPI, and $\triangle E I$. See the Appendix for the variable definitions. The principal components are extracted as explained in Table 3 and the text using the Connor and Korajczyk (1988) cross-sectional method. Panel A reports ten canonical correlations for each decade, sorted in descending order by their estimated squares. Corresponding Newey-West T-statistics (ten lags) for the correlations are also reported. Panel B summarizes significance levels for factor candidates. The following procedure is implemented to derive the significance levels of each factor candidate: First, for each canonical pair, the eigenvector weights for the 10 CK PCs are taken and the weighted average CK PC, (which is the canonical variate for the $10 \mathrm{CK}$ PCs that produced the canonical correlation for this particular pair) is constructed. Then, a regression using each CK PC canonical variate as the dependent variable and the candidate factor realizations as 15 independent variables is run over the sample months, 120 months for the last four decades and slightly fewer for the first decade. ${ }^{28}$ The square root of the R-square from this regression (not the adjusted R-square) is the canonical correlation. The coefficients of the regression are equal (after proper normalization) to the eigenvector's elements for the candidate factors. The tstatistics from the regression then give the significance level of each candidate factor. There are 10 pairs of canonical variates in each decade and a canonical correlation for each one; thus, there is a total of 50 such regressions. In Panel $\mathrm{B}$, the $1^{\text {st }}$ row presents the mean t-statistic over all canonical correlations. The $2^{\text {nd }}$ row reports the mean t-statistic when the canonical correlation itself is statistically significant. Rows\#3 to \#7 give the number of significant canonical correlation in each decade, respectively, and the bottom row (8) reports its average over the five decades. Critical rejection levels for the T-Statistic are 1.65 (10\%), 1.96 (5\%), and 2.59 (1\%). TStatistics breaching the $5 \%(1 \%)$ critical level are in boldface (boldface italic.)

(Table continued on next page)

\footnotetext{
${ }^{28}$ One of the factors is missing for the first 36 months.
} 
Panel A: Canonical correlations

1968-1974

1975-1984

1985-1994

1995-2004

2005-2014

\begin{tabular}{|c|c|c|c|c|c|c|c|c|c|c|}
\hline $\begin{array}{c}\text { Canonical } \\
\text { Variate }\end{array}$ & $\begin{array}{c}\text { Canonical } \\
\text { Correlation }\end{array}$ & T-Stat & $\begin{array}{c}\text { Canonical } \\
\text { Correlation }\end{array}$ & T-Stat & $\begin{array}{c}\text { Canonical } \\
\text { Correlation }\end{array}$ & T-Stat & $\begin{array}{c}\text { Canonical } \\
\text { Correlation }\end{array}$ & T-Stat & $\begin{array}{c}\text { Canonical } \\
\text { Correlation }\end{array}$ & T-Stat \\
\hline 1 & 0.999 & 23.904 & 0.998 & 27.236 & 0.995 & 24.155 & 0.989 & 25.589 & 0.997 & 29.212 \\
\hline 2 & 0.967 & 14.189 & 0.906 & 13.624 & 0.869 & 11.969 & 0.943 & 16.668 & 0.913 & 17.800 \\
\hline 3 & 0.885 & 8.849 & 0.833 & 8.472 & 0.727 & 7.797 & 0.791 & 10.390 & 0.858 & 13.372 \\
\hline 4 & 0.779 & 5.538 & 0.649 & 4.027 & 0.705 & 5.606 & 0.760 & 7.526 & 0.756 & 9.513 \\
\hline 5 & 0.641 & 3.415 & 0.602 & 2.003 & 0.566 & 2.997 & 0.633 & 4.300 & 0.746 & 6.874 \\
\hline 6 & 0.560 & 2.454 & 0.428 & -0.218 & 0.522 & 1.640 & 0.549 & 2.253 & 0.546 & 3.325 \\
\hline 7 & 0.530 & 1.930 & 0.409 & -0.851 & 0.422 & 0.195 & 0.387 & 0.562 & 0.514 & 1.992 \\
\hline 8 & 0.508 & 1.334 & 0.261 & -1.901 & 0.357 & -0.648 & 0.363 & 0.299 & 0.385 & 0.299 \\
\hline 9 & 0.390 & 0.413 & 0.228 & -1.610 & 0.283 & -1.351 & 0.307 & -0.091 & 0.298 & -0.438 \\
\hline 10 & 0.344 & 0.290 & 0.185 & -1.163 & 0.125 & -2.050 & 0.246 & -0.249 & 0.221 & -0.632 \\
\hline
\end{tabular}

Panel B: Significance levels for factor candidates

Factor candidates

\begin{tabular}{|c|c|c|c|c|c|c|c|c|c|c|c|c|c|c|c|}
\hline & \multirow[b]{2}{*}{$\begin{array}{c}\text { Rm- } \\
\text { Rf }\end{array}$} & \multicolumn{14}{|c|}{ Factor candidates } \\
\hline & & SMB & HML & $\begin{array}{l}\text { RM } \\
\text { W }\end{array}$ & CMA & $\mathrm{RF}$ & MOM & $\begin{array}{l}\mathrm{ST}_{-} \\
\mathrm{Rev}\end{array}$ & $\begin{array}{l}\mathrm{LT}_{-} \\
\mathrm{Rev}\end{array}$ & $\Delta \mathrm{DP}$ & $\Delta \mathrm{IP}$ & $\Delta \mathrm{TS}$ & UNEXPI & $\Delta \mathrm{EI}$ & LIQ \\
\hline Mean_t & 5.787 & 3.921 & 2.034 & 1.519 & 1.357 & 1.320 & 1.950 & 1.332 & 1.634 & 1.640 & 1.138 & 1.512 & 1.602 & 1.304 & 1.368 \\
\hline $\begin{array}{l}\text { Mean_t of } \\
\text { significant } \\
\text { Canonical } \\
\text { Corr }\end{array}$ & 8.649 & 5.616 & 2.924 & 2.002 & 1.561 & 1.809 & 2.455 & 1.596 & 2.066 & 2.141 & 1.384 & 1.940 & 2.064 & 1.535 & 1.749 \\
\hline Decade \# & & & & & & & Numb & of t-sta & $1.96 \mathrm{o}$ & of $10 \mathrm{fc}$ & ach dec & & & & \\
\hline 1 & 4 & 4 & 5 & 4 & 2 & 3 & 2 & 3 & 3 & 2 & 3 & 4 & 1 & 1 & 4 \\
\hline 2 & 3 & 2 & 4 & 1 & 3 & 1 & 2 & 3 & 4 & 1 & 2 & 2 & 5 & 1 & 2 \\
\hline 3 & 3 & 4 & 4 & 2 & 3 & 1 & 3 & 1 & 1 & 2 & 1 & 3 & 3 & 2 & 3 \\
\hline 4 & 6 & 4 & 5 & 3 & 3 & 2 & 5 & 3 & 2 & 5 & 1 & 4 & 4 & 3 & 4 \\
\hline 5 & 3 & 2 & 4 & 3 & 2 & 4 & 4 & 4 & 3 & 2 & 4 & 2 & 3 & 3 & 3 \\
\hline Mean & 3.8 & 3.2 & 4.2 & 3.0 & 2.0 & 2.2 & 3.6 & 2.4 & 2.6 & 3.6 & 1.4 & 2.8 & 3.4 & 1.6 & 2.0 \\
\hline
\end{tabular}


Table 5

\section{Summary Statistics for the Candidate Factor Betas and Characteristics}

Here are summary statistics for betas of the nine factors that pass our necessary condition, namely, Rm-Rf, SMB, HML, RMW, MOM, LT_REV, $\triangle \mathrm{DP}, \triangle \mathrm{TS}$ and UNEXPI. Betas (OLS slope coefficients) are computed for each individual stock in a multiple regression of the stock's monthly return on monthly factor realizations, using all observations available for each stock. To be included, a stock must have at least 24 monthly observations. The summary statistics are for the cross-stock distributions of each beta. Also included here are corresponding summary statistics for individual stock characteristics (defined in the Appendix). The sample period spans 564 months from January 1968 through December 2014. The first 36 months are lost due to the lagged -13 to -36 return, "Lag13-36."

\begin{tabular}{lccccccc} 
& Mean & Median & Sigma & Skewness & Kurtosis & Maximum & Minimum \\
\hline Rm-Rf & 0.927 & 0.929 & 0.031 & 0.026 & -0.658 & 0.994 & 0.861 \\
SMB & 0.800 & 0.808 & 0.045 & -0.911 & 1.069 & 0.873 & 0.647 \\
HML & 0.151 & 0.153 & 0.067 & -0.132 & -0.874 & 0.276 & 0.023 \\
RMW & 0.083 & 0.089 & 0.061 & -0.118 & -0.474 & 0.218 & -0.044 \\
MOM & -0.111 & -0.108 & 0.031 & -0.180 & -1.124 & -0.055 & -0.170 \\
LT_Rev & -0.020 & -0.017 & 0.018 & -0.145 & -0.309 & 0.031 & -0.060 \\
DDP & -1.009 & -1.028 & 0.379 & 0.162 & -1.190 & -0.269 & -1.709 \\
$\Delta$ TS & -0.447 & -0.433 & 0.179 & 0.003 & -1.145 & -0.141 & -0.805 \\
UNEXPI & 0.435 & 0.474 & 0.192 & -0.341 & -1.259 & 0.703 & 0.112 \\
RetLag1 & 1.069 & 1.503 & 5.426 & -0.465 & 3.232 & 27.338 & -27.660 \\
Lag2_12 & 0.351 & 0.559 & 1.823 & -0.518 & 0.932 & 5.866 & -6.483 \\
Lag13_36 & 0.437 & 0.567 & 1.134 & -0.596 & 0.689 & 3.252 & -3.342 \\
SizeLag1 & 11.88 & 11.62 & 0.904 & 0.580 & -0.892 & 13.810 & 10.385 \\
Book/Mkt & 0.874 & 0.797 & 0.319 & 1.722 & 4.112 & 2.221 & 0.453 \\
ProfRato & 0.274 & 0.208 & 0.457 & 6.199 & 38.34 & 3.332 & -0.124 \\
AsstGrth & 0.170 & 0.174 & 0.054 & -0.115 & 0.390 & 0.405 & 0.035 \\
\hline
\end{tabular}


Table 6

\section{Estimated Risk Premiums for Factors Candidates that Satisfy the Necessary Conditions}

Risk Premiums (in \%/month) are estimated from cross-sectional regressions computed using individual stock returns from 1968-2014 as dependent variables and, as explanatory variables, decile-sorted portfolio betas of the nine factors that pass necessary conditions including Rm-Rf, SMB, HML, RMW, MOM, LT_REV, $\triangle \mathrm{DP}, \triangle \mathrm{TS}$ and UNEXPI in Models 1 and 2, and the nine factors that pass necessary conditions and associated characteristics in Models 3 and 4. Characteristics include RetLag1, Lag2_12, Lag13_36, Size, Book/Mkt, ProfRato, and AsstGrth. See variable definitions in the Appendix. These selected factors are those that are significantly related to any canonical variate in all decades or that have mean t-statistics in the second row of Table 4 Panel B that exceed the one-tailed, 2.5\% cutoff based on the Chi-square value and an average number of significant t-stats exceeding 2.5 (see the bottom row of Table 4 Panel B). For the non-EIV estimation, OLS multiple regressions are run for each stock on all (nine) factors using all available observations for that stock. For the EIV calculations, stocks are sorted into ten groups (deciles) by market capitalization (Size), annually at the end of each June, based on NYSE size decile breakpoints. Then within each Size decile, stocks are sorted further by the OLS betas of the first factor (Rm-Rf) into ten deciles, thus resulting in 100 Size/first factor beta groups. Within each of the 100 groups, the equal-weighted average first factor beta of the group is assigned to each stock within that group. For each of the other eight factors, this procedure is repeated independently; ultimately, each stock's beta (for all nine betas) is replaced by the equalweighted portfolio beta of the double sorted size/beta group to which the stock belongs. This same procedure is redone every June, 1965-2014; then cross-sectional regressions are calculated in the 12 subsequent months of individual stock returns on the double-sorted portfolios betas (six months only after the 2014 sort.) The time series average over all months of the cross-sectional coefficients, termed the "risk premiums, " along with associated sampling statistics, are reported in the table. Critical rejections levels for the T-Statistic are $1.65(10 \%), 1.96(5 \%)$, and $2.59(1 \%)$. T-Statistics breaching the $5 \%(1 \%)$ critical level are in boldface (boldface italic.)

(Table continued on next page) 


\begin{tabular}{|c|c|c|c|c|c|c|c|c|}
\hline & \multicolumn{2}{|c|}{$\begin{array}{l}\text { No EIV correction } \\
\text { (Model 1) }\end{array}$} & \multicolumn{2}{|c|}{$\begin{array}{l}\text { EIV correction } \\
(\text { Model } 2)\end{array}$} & \multicolumn{2}{|c|}{$\begin{array}{l}\text { No EIV correction } \\
\text { (Model 3) }\end{array}$} & \multicolumn{2}{|c|}{$\begin{array}{l}\text { EIV correction } \\
\text { (Model 4) }\end{array}$} \\
\hline & Mean & T-Stat & Mean & T-Stat & Mean & T-Stat & Mean & T-Stat \\
\hline Constant & 0.600 & 9.467 & 0.603 & 6.895 & 2.211 & 9.014 & 1.829 & 5.726 \\
\hline Rm-Rf & 0.479 & 2.503 & 0.435 & 2.872 & 0.445 & 2.214 & 0.347 & 2.713 \\
\hline SMB & 0.036 & 0.276 & 0.049 & 0.486 & -0.240 & -1.823 & -0.077 & -0.962 \\
\hline HML & -0.184 & -1.484 & -0.127 & -1.456 & -0.371 & -2.843 & -0.247 & -3.613 \\
\hline RMW & 0.244 & 2.686 & 0.190 & 2.919 & 0.250 & 2.513 & 0.098 & 2.057 \\
\hline MOM & 0.480 & 2.606 & 0.264 & 1.875 & 0.303 & 1.620 & 0.190 & 1.653 \\
\hline LT_Rev & -0.197 & -1.827 & -0.113 & -1.408 & -0.259 & -2.334 & -0.112 & -1.645 \\
\hline$\Delta \overline{D P}$ & 0.016 & 1.457 & 0.014 & 1.659 & 0.025 & 2.104 & 0.017 & 2.211 \\
\hline$\Delta \mathrm{TS}$ & 0.049 & 1.316 & 0.058 & 1.952 & 0.063 & 1.576 & 0.065 & 2.462 \\
\hline UNEXPI & -0.087 & -2.702 & -0.074 & -2.801 & -0.083 & -2.365 & -0.027 & -1.189 \\
\hline RetLag1 & & & & & -0.062 & -21.89 & -0.060 & -19.07 \\
\hline Lag2_12 & & & & & 0.106 & 9.274 & 11.759 & 8.632 \\
\hline Lag13_36 & & & & & 0.038 & 2.878 & 2.141 & 1.362 \\
\hline SizeLag1 & & & & & -0.130 & -7.444 & -0.103 & -4.100 \\
\hline Book/Mkt & & & & & 0.210 & 6.267 & 0.176 & 4.256 \\
\hline ProfRato & & & & & 0.044 & 2.038 & 0.262 & 5.619 \\
\hline AsstGrth & & & & & -0.272 & -6.832 & -0.337 & -6.403 \\
\hline RSquare & & & 0.082 & & 0.124 & & 0.084 & \\
\hline SamplSize & & & 2839 & & 2688 & & 1706 & \\
\hline
\end{tabular}


Table 7

\section{Returns of Hedge Portfolios Associated with Factors that Satisfy Necessary Conditions}

Here are summary statistics for returns (in \%/month) of hedge portfolios associated with the nine factors that satisfy the necessary conditions in Table 4. The factors include Rm-Rf, SMB, HML, RMW, MOM, LT_REV, $\triangle \mathrm{DP}, \triangle \mathrm{TS}$, and UNEXPI. For each candidate factor, hedge portfolios are formed by a long position in a group of stocks with the highest betas on the factor and a short position a group with the lowest betas; this is done with replacement. Panel A (C) shows the returns of the hedge portfolios with the top and bottom deciles (the top 30\% and bottom $30 \%$ ). Panel B (D) show the excess returns of the market (Rm-Rf) and augmented returns, which is the hedge portfolio return added to the market excess return (Rm-Rf). The augmented return of $\mathrm{Rm}-\mathrm{Rf}$ is itself appended with its own hedge portfolio. The other eight to the right of it are for the other eight hedge portfolios. The Sharpe ratio (Sharpe) and a t-statistic of the Sharpe ratio against 0.6 (Sharpe t), the MacKinlay (1995) threshold, are also reported. Critical rejections levels for the T-Statistic are $1.65(10 \%), 1.96(5 \%)$, and $2.59(1 \%)$. T-Statistics breaching the 5\% (1\%) critical level are in boldface (boldface italic.)

Panel A: Hedge portfolio from 10\% top and bottom

\begin{tabular}{lccccccccc} 
& Rm-Rf & SMB & HML & RMW & MOM & LT_Rev & $\Delta$ DP & $\Delta$ TS & UNEXPI \\
\hline Mean & 0.691 & -0.107 & -0.415 & 0.667 & 0.360 & -0.035 & 0.504 & 0.875 & -0.994 \\
Std. Dev & 6.559 & 6.411 & 6.264 & 5.873 & 5.604 & 5.029 & 5.335 & 5.663 & 5.943 \\
t(Mean) & $\mathbf{2 . 5 0 2}$ & -0.395 & -1.572 & $\mathbf{2 . 6 9 8}$ & 1.527 & -0.164 & $\mathbf{2 . 2 4 5}$ & $\mathbf{3 . 6 7 0}$ & $\mathbf{- 3 . 9 7 1}$ \\
\hline
\end{tabular}

Panel B: Market return plus 10\% top and bottom hedge portfolio returns

\begin{tabular}{|c|c|c|c|c|c|c|c|c|c|c|}
\hline & \multirow{2}{*}{$\begin{array}{c}\text { Rm- } \\
\text { Rf }\end{array}$} & \multicolumn{8}{|c|}{ Augmented returns } & \multirow[b]{2}{*}{ UNEXPI } \\
\hline & & $\mathrm{Rm}-\mathrm{Rf}$ & SMB & HML & RMW & MOM & LT_Rev & $\Delta \mathrm{DP}$ & $\Delta \mathrm{TS}$ & \\
\hline Mean & 0.490 & 1.181 & 0.383 & 0.075 & 1.157 & 0.850 & 0.455 & 0.995 & 1.365 & -0.504 \\
\hline Std. Dev & 4.584 & 10.739 & 8.711 & 7.191 & 7.140 & 6.595 & 7.032 & 7.207 & 7.200 & 7.821 \\
\hline $\mathrm{t}($ Mean $)$ & 2.539 & 2.612 & 1.045 & 0.249 & 3.849 & 3.062 & 1.538 & 3.277 & 4.503 & -1.529 \\
\hline Sharpe & 0.370 & 0.381 & 0.152 & 0.036 & 0.561 & 0.447 & 0.224 & 0.478 & 0.657 & 0.223 \\
\hline Sharpe t & -5.275 & -5.02 & -10.57 & -13.38 & -0.850 & -3.472 & -8.811 & -2.744 & 1.225 & -8.842 \\
\hline
\end{tabular}

Panel C: Hedge portfolio 30\% top and bottom

\begin{tabular}{cccccccccc} 
& Rm- & & & & & & & \\
& Rf & SMB & HML & RMW & MOM & LT_Rev & $\Delta$ DP & $\Delta$ TS & UNEXPI \\
\hline Mean & 0.368 & -0.154 & -0.122 & 0.522 & 0.246 & 0.015 & 0.281 & 0.609 & -0.664 \\
Std. Dev & 4.327 & 4.433 & 4.046 & 3.734 & 3.488 & 2.989 & 3.178 & 3.666 & 3.867 \\
t(Mean) & $\mathbf{2 . 0 1 7}$ & -0.824 & -0.715 & $\mathbf{3 . 3 2 1}$ & 1.675 & 0.123 & $\mathbf{2 . 1 0 2}$ & $\mathbf{3 . 9 4 5}$ & $\mathbf{- 4 . 0 8 0}$ \\
\hline
\end{tabular}

Panel D: Market return plus 30\% top-and-bottom hedge portfolio return

\begin{tabular}{|c|c|c|c|c|c|c|c|c|c|c|}
\hline & \multirow{2}{*}{$\mathrm{Rm}-\mathrm{Rf}$} & \multicolumn{8}{|c|}{ Augmented returns } & \multirow[b]{2}{*}{ UNEXPI } \\
\hline & & Rm-Rf & SMB & HML & RMW & MOM & LT_Rev & $\Delta \mathrm{DP}$ & $\Delta \mathrm{TS}$ & \\
\hline Mean & 0.490 & 0.858 & 0.336 & 0.368 & 1.012 & 0.736 & 0.506 & 0.771 & 1.099 & -0.174 \\
\hline Std. Dev & 4.584 & 8.669 & 7.335 & 5.319 & 5.589 & 4.920 & 5.756 & 5.540 & 5.588 & 6.469 \\
\hline $\mathrm{t}($ Mean $)$ & 2.539 & 2.349 & 1.089 & 1.644 & 4.302 & 3.554 & 2.086 & 3.307 & 4.672 & -0.640 \\
\hline Sharpe & 0.370 & 0.343 & 0.159 & 0.240 & 0.627 & 0.518 & 0.304 & 0.482 & 0.681 & 0.093 \\
\hline Sharpe t & -5.275 & -5.939 & -10.41 & -8.433 & 0.596 & -1.821 & -6.865 & -2.644 & 1.743 & -12.007 \\
\hline
\end{tabular}


Table 8

\section{Returns of Hedge Portfolios Associated with Characteristics}

Here are summary statistics for returns (in \%/month) on hedge portfolios associated with the seven characteristics RetLag1, Lag2_12, Lag13_36, Size, Book/Mkt, ProfRato, and AsstGrth. See variable definitions in the Appendix. For each characteristic, hedge portfolios are formed by a long position in a group of the stocks with high values of the characteristic and a short position a group with low characteristic values; this is done with replacement. Panel A (C) shows the returns of the hedge portfolios with the top and bottom deciles (the top 30\% and bottom 30\%). Panel B (D) show the returns of market (Rm-Rf) and augmented returns, which is the hedged portfolio returns added to the market return (Rm-Rf). The seven to the right of it are for the characteristics. The Sharpe ratio (Sharpe) and t-statistic of the Sharpe ratio against 0.6, (Sharpe t), the MacKinlay (1995) threshold, are reported. Critical rejections levels for the T-Statistic are 1.65 (10\%), 1.96 $(5 \%)$, and $2.59(1 \%)$. T-Statistics breaching the 5\% (1\%) critical level are in boldface (boldface italic.)

Panel A: Hedge portfolio from 10\% top and bottom

\begin{tabular}{lccccccc} 
& RetLag1 & Lag2_12 & Lag13_36 & SizeLag1 & Book/Mkt & ProfRato & AsstGrth \\
\hline Mean & -1.791 & 1.814 & 0.009 & 0.024 & 0.797 & 0.904 & -0.510 \\
Std. Dev & 4.157 & 5.247 & 4.445 & 5.358 & 3.824 & 3.932 & 2.603 \\
t(Mean) & $\mathbf{- 1 0 . 2 3}$ & $\mathbf{8 . 2 1 2}$ & 0.046 & 0.105 & $\mathbf{4 . 9 5 1}$ & $\mathbf{5 . 4 5 8}$ & $\mathbf{- 4 . 6 5 1}$ \\
\hline
\end{tabular}

Panel B: Market return plus 10\% top and bottom hedge portfolio returns

\begin{tabular}{lcccccccc} 
& Rm- & \multicolumn{7}{c}{ Augmented returns } \\
\cline { 3 - 9 } & Rf & RetLag1 & Lag2_12 & Lag13_36 & SizeLag1 & Book/Mkt & ProfRato & AsstGrth \\
\hline Mean & 0.490 & -1.300 & 2.304 & 0.499 & 0.514 & 1.287 & 1.394 & -0.020 \\
Std. Dev & 4.584 & 5.255 & 6.775 & 6.819 & 7.333 & 4.859 & 5.788 & 5.951 \\
t(Mean) & 2.539 & -5.877 & 8.077 & 1.737 & 1.664 & 6.292 & 5.719 & -0.079 \\
Sharpe & 0.370 & 0.857 & 1.178 & 0.253 & 0.243 & 0.918 & 0.834 & 0.011 \\
Sharpe t & $\mathbf{- 5 . 2 7 5}$ & $\mathbf{5 . 2 2 3}$ & $\mathbf{1 0 . 5 5}$ & $\mathbf{- 8 . 1 0 4}$ & $\mathbf{- 8 . 3 6 3}$ & $\mathbf{6 . 3 3 1}$ & $\mathbf{4 . 7 9 1}$ & $\mathbf{- 1 3 . 9 8}$ \\
\hline
\end{tabular}

Panel C: Hedge portfolio 30\% top and bottom

\begin{tabular}{lccccccc} 
& RetLag1 & Lag2_12 & Lag13_36 & SizeLag1 & Book/Mkt & ProfRato & AsstGrth \\
\hline Mean & -1.011 & 1.040 & -0.035 & 0.097 & 0.562 & 0.420 & -0.347 \\
Std. Dev & 2.870 & 3.643 & 3.032 & 3.958 & 2.643 & 2.533 & 1.738 \\
t(Mean) & $\mathbf{- 8 . 3 6 8}$ & $\mathbf{6 . 7 7 8}$ & -0.278 & 0.583 & $\mathbf{5 . 0 4 5}$ & $\mathbf{3 . 9 4 0}$ & $\mathbf{- 4 . 7 4 7}$ \\
\hline
\end{tabular}

Panel D: Market return plus 30\% top-and-bottom hedge portfolio return

\begin{tabular}{lcccccccc} 
& \multirow{2}{*}{ Rm-Rf } & \multicolumn{7}{c}{ Augmented returns } \\
\cline { 3 - 8 } & & RetLag1 & Lag2_12 & Lag13_36 & SizeLag1 & Book/Mkt & ProfRato & AsstGrth \\
\hline Mean & 0.490 & -0.521 & 1.530 & 0.455 & 0.587 & 1.052 & 0.910 & 0.143 \\
Std. Dev & 4.584 & 4.640 & 5.562 & 5.591 & 6.302 & 4.330 & 5.094 & 5.505 \\
t(Mean) & 2.539 & -2.666 & 6.533 & 1.931 & 2.213 & 5.768 & 4.244 & 0.616 \\
Sharpe & 0.370 & 0.389 & 0.953 & 0.282 & 0.323 & 0.841 & 0.619 & 0.090 \\
Sharpe t & $\mathbf{- 5 . 2 7 5}$ & $\mathbf{- 4 . 8 3 3}$ & $\mathbf{6 . 9 5 0}$ & $\mathbf{- 7 . 4 1 4}$ & $\mathbf{- 6 . 4 1 6}$ & $\mathbf{4 . 9 2 6}$ & 0.414 & $\mathbf{- 1 2 . 0 9}$ \\
\hline
\end{tabular}


Table 9

Correlations between Factor- and Characteristic-Based Hedge Portfolios

Here are correlations between the hedge portfolios corresponding to the nine factors that pass necessary conditions and the hedge portfolios corresponding to the seven characteristics. These are hedge portfolios that are long the largest $30 \%$ of the values and short the smallest $30 \%$ for each factor and characteristic. The construction of the hedge portfolios is explained in Tables 7 and 8. See the Appendix for variable definitions. Correlations that are greater than or equal to 0.5 in absolute value are in boldface.

\begin{tabular}{lccccccccc} 
& Rm-Rf & SMB & HML & RMW & MOM & LT_Rev & $\Delta$ DP & $\Delta$ TS & UNEXPI \\
\hline RetLag1 & -0.302 & -0.305 & 0.117 & 0.049 & 0.437 & -0.140 & 0.154 & 0.195 & -0.288 \\
Lag2_12 & -0.115 & -0.304 & -0.011 & 0.114 & $\mathbf{0 . 7 4 5}$ & -0.047 & 0.148 & 0.309 & -0.280 \\
Lag13_36 & 0.026 & -0.343 & -0.143 & 0.143 & 0.263 & -0.307 & 0.175 & 0.388 & -0.309 \\
SizeLag1 & 0.145 & $\mathbf{- 0 . 6 4 2}$ & -0.092 & 0.175 & 0.366 & -0.045 & 0.231 & $\mathbf{0 . 6 3 9}$ & $\mathbf{- 0 . 5 2 2}$ \\
Book/Mkt & -0.363 & 0.108 & $\mathbf{0 . 6 4 3}$ & 0.199 & -0.006 & 0.078 & -0.088 & -0.142 & -0.036 \\
ProfRato & -0.032 & $\mathbf{- 0 . 5 0 8}$ & 0.230 & $\mathbf{0 . 6 1 1}$ & 0.319 & -0.034 & 0.248 & $\mathbf{0 . 5 8 5}$ & $\mathbf{- 0 . 5 9 3}$ \\
AsstGrth & 0.390 & 0.023 & -0.353 & 0.088 & -0.059 & -0.140 & 0.118 & 0.149 & -0.067 \\
\hline
\end{tabular}




\section{Internet Appendix A}

This Internet Appendix shows the results of some robustness checks on our simulation. First, we apply randomly selected portfolios as candidate factors in Table A1. Each of these portfolios are based on going long-short on randomly selected deciles. While we use the case of 6,000 stocks, the results do not substantially differ for a smaller sample. The fifty random portfolios each pass less than $10 \%$ of the time; the passing rate is mostly between four and six percent. Second, we consider the passing rate of a factor that involves replacing $10 \%$ of the sample in each period and of another factor that is a randomly selected stock in Table A2. We find that the passing rate of the factor with random replacement is less than $0.05 \%$. Our analysis indicates that changing the replacement rate to $20 \%$ per period does not materially alter the pass rate. The randomly picked stock passes the first stage less than $10 \%$ of the time when the standard deviation of measurement errors is $1 \%$ and virtually never when it is $0.25 \%$. Third, we apply as a candidate factor a linear combination (average) of the first two factors in Table A3. The motivation for the latter is that we may not observe factors individually in reality. This candidate passes the sufficient condition more than $90 \%$ of the time. 
Table A1

\section{Passing Rates (Necessary Condition) of Random Factors}

This table presents the percentage passing rate of candidate factors from 5,000 simulations. f.1 to f.5 are factors that are associated with risk premiums. Factors g.1, g.2, and g. 3 do not command risk premiums. $\mathrm{Zi}(\mathrm{i}=1, \ldots .5)$ represents five characteristics. f.1 has a mean and standard deviation equal to those of the excess return on the value-weighted CRSP index. The factors f.2 to f.5 are generated independently from a normal distribution with the actual mean and standard deviation of HML. Factors g have mean zero, with standard deviation equal to that of HML. The five characteristics represent firm-specific information that varies across time, and they are generated at each time period from the uniform distribution $[-0.1 \%, 0.1 \%]$. The standard deviation for the error term is computed to ensure that for a "typical" stock, whose betas equal their cross-sectional means, the $R^{2}$ from a regression of individual stock returns on the factors equals $25 \%$. Random factors a are long-short portfolios where $10 \%$ of all stocks are randomly selected and longed and another $10 \%$ of all stocks are randomly chosen and shorted. This table shows the passing rates for the fifty a factors. The factors that pass the first stage are those that are significantly related to any canonical variate or that have mean t-statistics in the second row of Table 4 Panel B in the manuscript that exceed the onetailed, $2.5 \%$ cutoff based on the Chi-square value of 1.96 . The simulation in this table is based on 6,000 stocks, 720 and 840 periods of time, and standard deviation of measurement errors $\eta_{i, t}$ and $\iota_{i, t}$ of $\tilde{f}_{i, t}$ and $\tilde{g}_{i, t}$, respectively of $1.00 \%$ and $0.25 \%$. Section 6 of the manuscript describes the Data Generating Process used for $\tilde{f}_{i, t}$ and $\tilde{g}_{i, t}$.

Standard deviation of measurement errors $=1.00 \%$

Standard deviation of measurement errors $=0.25 \%$

\begin{tabular}{|c|c|c|c|c|}
\hline Factor candidates & Periods of time $=720$ & Periods of time $=840$ & Periods of time $=720$ & Periods of time $=840$ \\
\hline a. 1 & 5.9 & 9.4 & 0.02 & 0.02 \\
\hline a. 2 & 5.9 & 8.7 & 0.02 & 0.04 \\
\hline a.3 & 5.7 & 8.5 & 0.00 & 0.00 \\
\hline a. 4 & 5.7 & 8.6 & 0.00 & 0.00 \\
\hline a. 5 & 5.6 & 9.6 & 0.00 & 0.00 \\
\hline a.6 & 5.9 & 8.9 & 0.02 & 0.02 \\
\hline a.7 & 5.9 & 8.1 & 0.02 & 0.10 \\
\hline a.8 & 6.1 & 9.1 & 0.00 & 0.02 \\
\hline a.9 & 6.0 & 9.2 & 0.00 & 0.00 \\
\hline a. 10 & 6.2 & 9.8 & 0.02 & 0.00 \\
\hline a.11 & 5.9 & 8.3 & 0.02 & 0.00 \\
\hline a.12 & 5.5 & 8.6 & 0.00 & 0.00 \\
\hline a.13 & 5.8 & 8.1 & 0.02 & 0.00 \\
\hline a. 14 & 5.7 & 8.6 & 0.02 & 0.00 \\
\hline a. 15 & 6.0 & 8.9 & 0.00 & 0.04 \\
\hline a.16 & 5.8 & 10 & 0.02 & 0.00 \\
\hline a. 17 & 6.0 & 9.0 & 0.02 & 0.04 \\
\hline
\end{tabular}


Standard deviation of measurement errors $=1.00 \%$

\begin{tabular}{|c|c|c|c|c|}
\hline \multirow[b]{2}{*}{ Factor candidates } & \multirow[b]{2}{*}{ Periods of time $=720$} & \multirow[b]{2}{*}{ Periods of time $=840$} & \multirow[b]{2}{*}{ Periods of time $=720$} & \multirow[b]{2}{*}{ Periods of time $=840$} \\
\hline & & & & \\
\hline a.18 & 5.6 & 9.2 & 0.04 & 0.00 \\
\hline a.19 & 5.5 & 9.3 & 0.00 & 0.00 \\
\hline a. 20 & 5.6 & 9.5 & 0.00 & 0.00 \\
\hline a. 21 & 5.5 & 8.7 & 0.00 & 0.00 \\
\hline a. 22 & 5.4 & 9.3 & 0.00 & 0.00 \\
\hline a. 23 & 6.3 & 9.3 & 0.02 & 0.04 \\
\hline a. 24 & 5.7 & 9.8 & 0.00 & 0.02 \\
\hline a. 25 & 5.7 & 7.9 & 0.02 & 0.00 \\
\hline a. 26 & 5.6 & 9.3 & 0.00 & 0.00 \\
\hline a. 27 & 6.0 & 8.6 & 0.00 & 0.00 \\
\hline a. 28 & 5.8 & 11 & 0.00 & 0.00 \\
\hline a. 29 & 5.7 & 9.5 & 0.00 & 0.04 \\
\hline a. 30 & 5.3 & 9.8 & 0.00 & 0.02 \\
\hline a. 31 & 5.1 & 8.1 & 0.00 & 0.04 \\
\hline a. 32 & 5.7 & 8.1 & 0.00 & 0.00 \\
\hline a. 33 & 5.7 & 9.0 & 0.04 & 0.00 \\
\hline a. 34 & 6.0 & 8.9 & 0.00 & 0.00 \\
\hline a. 35 & 5.9 & 8.3 & 0.00 & 0.02 \\
\hline a.36 & 6.1 & 8.1 & 0.00 & 0.02 \\
\hline a. 37 & 5.5 & 9.4 & 0.00 & 0.00 \\
\hline a. 38 & 5.8 & 8.8 & 0.00 & 0.08 \\
\hline a.39 & 6.1 & 9.0 & 0.04 & 0.02 \\
\hline a. 40 & 5.8 & 10 & 0.02 & 0.00 \\
\hline a. 41 & 5.7 & 9.6 & 0.00 & 0.02 \\
\hline a. 42 & 5.6 & 11 & 0.00 & 0.00 \\
\hline a. 43 & 5.7 & 8.0 & 0.00 & 0.00 \\
\hline a. 44 & 5.7 & 8.6 & 0.00 & 0.02 \\
\hline a. 45 & 5.7 & 10 & 0.00 & 0.00 \\
\hline a. 46 & 6.0 & 8.8 & 0.00 & 0.04 \\
\hline a. 47 & 5.9 & 9.5 & 0.00 & 0.00 \\
\hline a. 48 & 5.3 & 9.7 & 0.02 & 0.00 \\
\hline a. 49 & 5.2 & 9.4 & 0.00 & 0.02 \\
\hline a. 50 & 5.8 & 9.0 & 0.06 & 0.02 \\
\hline
\end{tabular}


Table A2

\section{The Passing Rate of the Necessary Condition of the Two New Random Factors}

This table presents the percentage passing rate of candidate factors from 5,000 simulations. f. 1 to f.5 are factors that are associated with risk premiums. Factors g.1, g.2, and g.3 do not command risk premiums. $\mathrm{Zi}(\mathrm{i}=1, \ldots .5)$ represents five characteristics. f.1 has mean and standard deviation equal to those of the excess return on the value-weighted CRSP index. The factors f.2 to f.5 are generated independently from a distribution with the actual mean and standard deviation of HML. Factors g.1, g.2, and g.3 do not command risk premiums and thus have mean zero, with standard deviation equal to that of HML. The five characteristics represent firm-specific information that varies across time, and they are generated at each time period from the uniform distribution $[-0.1 \%, 0.1 \%]$. The standard deviation for the error term is computed to ensure that, for a "typical" stock whose betas equal their cross-sectional means, the $R^{2}$ from a regression of individual stock returns on the factors equals $25 \%$. Factor new.a1 is constructed by going long on randomly chosen $10 \%$ of stocks and shorting another randomly chosen $10 \%$ of all stocks in the first period, and randomly replacing $10 \%$ of the long leg and $10 \%$ of the short leg in subsequent periods. The return on each portfolio in each period is the new.a1. The factor "new.a2" represents the return on a single randomly selected stock. The selected factors are those that are significantly related to any canonical variate or that have mean t-statistics in the second row of Table 4 Panel B of the manuscript that exceed the one-tailed, $2.5 \%$ cutoff based on the Chi-square value of 1.96. The simulation in this table is based on 6,000 stocks, 720 and 840 periods of time, and standard deviation of measurement errors $\eta_{i, t}$ and $\iota_{i, t}$ of $\tilde{f}_{i, t}$ and $\tilde{g}_{i, t}$, respectively of $1.00 \%$ and $0.25 \%$. Section 6 of the manuscript describes the Data Generating Process used for $\tilde{f}_{i, t}$ and $\tilde{g}_{i, t}$.

Standard deviation of measurement errors $=1.00 \%$

Factor candidates
Standard deviation of measurement errors $=0.25 \%$

\begin{tabular}{ccccc}
\hline new.a1 & 0.00 & 0.02 & 0.00 & 0.00 \\
new.a2 & 9.6 & 8.6 & 0.00 & 0.00 \\
\hline
\end{tabular}


Table A3

\section{Simulation Results with f.new}

This table presents the percentage pass rates (1st column) for the necessary condition (Columns (1)) and the sufficient condition (Columns (2)) from 5,000 simulations. f.new to f.5 are factors that are associated with risk premiums where f.new is the average of f. 1 and f.2 plus $\eta_{t}$ (see Section 6 of the manuscript). f. 1 and f.2 are defined in Tables A1 and A2. Z represents five characteristics. f.1 has mean and standard deviation equal to those of the excess return on the value-weighted CRSP index. The factors f.2 to f.5 are generated independently from a distribution with the actual mean and standard deviation of HML. Factors g.1, g.2, and g.3 are independent of each other and diversifiable and thus have mean zero, with standard deviations equal to that of HML. The five characteristics represent firm-specific information that varies across time, and they are generated at each time period from the uniform distribution [-0.1\%, $0.1 \%]$. The standard deviation for the error term is computed to ensure that for a "typical" stock whose betas equal their cross-sectional means the $R^{2}$ from a regression of individual stock returns on the factors equals $25 \%$. The simulation is based on 6,000 stocks, 720 periods and 840 periods, and standard deviation of measurement errors $\eta_{i, t}$ and $\iota_{i, t}$ of $\tilde{f}_{i, t}$ and $\tilde{g}_{i, t}$, respectively of $1.00 \%$ and $0.25 \%$. Section 6 of the manuscript describes the Data Generating Process used for $\tilde{f}_{i, t}$ and $\tilde{g}_{i, t}$.

Standard deviation of measurement errors $=1.00 \%$

\begin{tabular}{|c|c|c|c|c|c|c|c|c|}
\hline \multirow{2}{*}{$\begin{array}{c}\text { Factor } \\
\text { Candidates }\end{array}$} & \multicolumn{2}{|c|}{ Periods of time $=720$} & \multicolumn{2}{|c|}{ Periods of time $=840$} & \multicolumn{2}{|c|}{ Periods of time $=720$} & \multicolumn{2}{|c|}{ Periods of time $=840$} \\
\hline & $\begin{array}{c}\text { Necessary } \\
\text { passing rate } \\
\text { (1) }\end{array}$ & $\begin{array}{l}\text { Significance } \\
\text { rate } \\
(2)\end{array}$ & $\begin{array}{c}\text { Necessary } \\
\text { passing rate } \\
\text { (1) }\end{array}$ & $\begin{array}{l}\text { Significance } \\
\text { rate } \\
\text { (2) }\end{array}$ & $\begin{array}{l}\text { Necessary } \\
\text { passing rate } \\
\text { (1) }\end{array}$ & $\begin{array}{c}\text { Significance } \\
\text { rate } \\
\text { (2) }\end{array}$ & $\begin{array}{l}\text { Necessary } \\
\text { passing rate } \\
\text { (1) }\end{array}$ & $\begin{array}{l}\text { Significance } \\
\text { rate } \\
(2)\end{array}$ \\
\hline f.new & 100 & 95 & 100 & 98 & 100 & 95 & 100 & 98 \\
\hline f.3 & 100 & 91 & 100 & 93 & 100 & 90 & 100 & 93 \\
\hline f. 4 & 100 & 90 & 100 & 94 & 100 & 90 & 100 & 93 \\
\hline f. 5 & 100 & 90 & 100 & 93 & 100 & 90 & 100 & 94 \\
\hline g.1 & 100 & 3.8 & 100 & 4.2 & 100 & 4.3 & 100 & 4.1 \\
\hline g.2 & 100 & 3.8 & 100 & 4.6 & 100 & 4.3 & 100 & 4.7 \\
\hline g. 3 & 100 & 3.2 & 100 & 4.9 & 100 & 3.6 & 100 & 4.1 \\
\hline a.1 / Z.1 & - & 100 & - & 100 & - & 100 & - & 100 \\
\hline a. 2 / Z.2 & - & 100 & - & 100 & - & 100 & - & 100 \\
\hline a. $3 / \mathrm{Z} .3$ & - & 100 & - & 100 & - & 100 & - & 100 \\
\hline a.4 / Z.4 & - & 100 & - & 100 & - & 100 & - & 100 \\
\hline a.5 / Z.5 & - & 100 & - & 100 & - & 100 & - & 100 \\
\hline
\end{tabular}

Standard deviation of measurement errors $=0.25 \%$

(1)




\section{Internet Appendix B \\ Results Including the Amihud Illiquidity Measure}

This Internet Appendix B presents the equivalents of Tables 6 through 9 in the paper for a limited sample that includes Amihud's illiquidity ratio as a characteristic. The Table 7 equivalent is omitted as it is unchanged relative to the one in the manuscript

\section{Table B-6}

\section{Estimated Risk Premiums for Factors Candidates that Satisfy the Necessary Conditions with a Sample Limited by the Inclusion of Amihud's Illiquidity Ratio}

The paper's Table 6, Models 3 and 4 are redone here using a sample limited by the inclusion of Amihud's illiquidity ratio (“Amihud".) Risk Premiums (in \%/month) are estimated from cross-sectional regressions computed using individual stock returns from 1968-2014 as dependent variables and, as explanatory variables, decile-sorted portfolio betas of the nine factors that pass necessary conditions including Rm-Rf, SMB, HML, RMW, MOM, LT_REV, $\triangle \mathrm{DP}, \Delta \mathrm{TS}$ and UNEXPI and associated characteristics in Models 3 and 4. Characteristics include RetLag1, Lag2_12, Lag13_36, Size, Book/Mkt, ProfRato, and AsstGrth. See variable definitions in the Appendix. These selected factors are those that are significantly related to any canonical variate in all decades or that have mean $\mathrm{t}$ statistics in the second row of Table 4 Panel B that exceed the one-tailed, 2.5\% cutoff based on the Chi-square value and an average number of significant t-stats exceeding 2.5 (see the bottom row of Table 4 Panel B). For the non-EIV estimation, OLS multiple regressions are run for each stock on all (nine) factors using all available observations for that stock. For the EIV calculations, stocks are sorted into ten groups (deciles) by market capitalization (Size), annually at the end of each June, based on NYSE size decile breakpoints. Then within each Size decile, stocks are sorted further by the OLS betas of the first factor (Rm-Rf) into ten deciles, thus resulting in $100 \mathrm{Size/first} \mathrm{factor} \mathrm{beta} \mathrm{groups.} \mathrm{Within} \mathrm{each} \mathrm{of} \mathrm{the} 100$ groups, the equal-weighted average first factor beta of the group is assigned to each stock within that group. For each of the other eight factors, this procedure is repeated independently; ultimately, each stock's beta (for all nine betas) is replaced by the equal-weighted portfolio beta of the double sorted size/beta group to which the stock belongs. This same procedure is redone every June, 1965-2014; then cross-sectional regressions are calculated in the 12 subsequent months of individual stock returns on the double-sorted portfolios betas (six months only after the 2014 sort.) The time series average over all months of the cross-sectional coefficients, termed the "risk premiums," along with associated sampling statistics, are reported in the table. Critical rejections levels for the T-Statistic are 1.65 (10\%), $1.96(5 \%)$, and 2.59 (1\%). T-Statistics breaching the $5 \%(1 \%)$ critical level are in boldface (boldface italic.)

(The table is continued on the next page) 
No EIV Correction (Model 3)

\begin{tabular}{|c|c|c|c|c|}
\hline & \multicolumn{2}{|c|}{ (Model 3) } & \multicolumn{2}{|c|}{ (Model 4) } \\
\hline & Mean & T-Stat & Mean & T-Stat \\
\hline Constant & 2.390 & 8.827 & 2.649 & 6.489 \\
\hline SMB & -0.320 & -2.522 & -0.161 & -2.391 \\
\hline HML & -0.395 & -3.077 & -0.190 & -3.210 \\
\hline RMW & 0.300 & 3.021 & 0.071 & 1.738 \\
\hline MOM & 0.426 & 2.260 & 0.189 & 1.832 \\
\hline LT_Rev & -0.301 & -2.788 & -0.088 & -1.470 \\
\hline$\Delta \overline{D P}$ & 0.025 & 2.106 & 0.020 & 2.820 \\
\hline$\Delta \mathrm{TS}$ & 0.039 & 0.992 & 0.057 & 2.474 \\
\hline UNEXPI & -0.103 & -2.929 & -0.018 & -0.915 \\
\hline RetLag1 & -0.060 & -19.831 & -0.056 & -15.933 \\
\hline Lag2_12 & 0.103 & 7.818 & 12.528 & 7.596 \\
\hline Lag13_36 & 0.032 & 2.396 & 0.626 & 0.375 \\
\hline SizeLag1 & -0.137 & -7.608 & -0.146 & -5.210 \\
\hline Book/Mkt & 0.218 & 5.360 & 0.131 & 2.152 \\
\hline ProfRato & 0.144 & 3.979 & 0.275 & 3.750 \\
\hline AsstGrth & -0.326 & -6.692 & -0.470 & -5.970 \\
\hline Amihud & -0.005 & -0.388 & -0.044 & -2.265 \\
\hline RSquare & \multicolumn{2}{|c|}{0.129} & \multicolumn{2}{|c|}{0.078} \\
\hline SamplSize & \multicolumn{2}{|c|}{1946} & \multicolumn{2}{|c|}{1171} \\
\hline
\end{tabular}


Table B-8

\section{Returns of Hedge Portfolios Associated with Characteristics with a Sample} Limited by the Inclusion of Amihud's Illiquidity Ratio

Table 8 is redone here using a sample limited by the inclusion of Amihud's illiquidity ratio. Here are summary statistics for returns (in \%/month) on hedge portfolios associated with the seven characteristics RetLag1, Lag2_12, Lag13_36, Size, Book/Mkt, ProfRato, and AsstGrth. See variable definitions in the Appendix. For each characteristic, hedge portfolios are formed by a long position in a group of the stocks with high values of the characteristic and a short position a group with low characteristic values; this is done with replacement. Panel A (C) shows the returns of the hedge portfolios with the top and bottom deciles (the top 30\% and bottom $30 \%$ ). Panel B (D) show the returns of market (Rm-Rf) and augmented returns, which is the hedged portfolio returns added to the market return (Rm-Rf). The Sharpe ratio and t-statistic of the Sharpe ratio against 0.6, the MacKinlay (1995) threshold, are reported. Critical rejections levels for the T-Statistic are $1.65(10 \%), 1.96(5 \%)$, and $2.59(1 \%)$. T-Statistics breaching the 5\% (1\%) critical level are in boldface (boldface italic.)

Panel A: Hedge portfolio from 10\% top and bottom

\begin{tabular}{lcccccccc} 
Month & RetLag1 & Lag2_12 & Lag13_36 & SizeLag1 & Book/Mkt & Profit & AsstGrth & Amihud \\
\hline Mean & -1.461 & 0.831 & -0.886 & -1.230 & -1.403 & 0.707 & -0.075 & 0.912 \\
Std. Dev & 3.954 & 5.122 & 3.675 & 4.006 & 4.993 & 3.436 & 2.876 \\
t(Mean) & $\mathbf{- 8 . 7 7 4}$ & $\mathbf{3 . 8 5 5}$ & $\mathbf{- 5 . 7 2 3}$ & $\mathbf{- 7 . 2 9 0}$ & $\mathbf{- 6 . 6 7 2}$ & $\mathbf{4 . 8 8 5}$ & -0.617 & $\mathbf{5 . 6 5 8}$ \\
\hline
\end{tabular}

Panel B: Market return plus 10\% top and bottom hedge portfolio returns

\begin{tabular}{lccccccccc} 
& & \multicolumn{9}{c}{ Augmented returns } \\
\cline { 2 - 9 } & Rm-Rf & RetLag1 & Lag2_12 & Lag13_36 & SizeLag1 & Book/Mkt & Profit & AsstGrth & Amihud \\
\hline Mean & 0.490 & -0.971 & 1.321 & -0.395 & -0.740 & -0.913 & 1.197 & 0.415 \\
Std. Dev & 4.584 & 5.060 & 6.708 & 6.330 & 6.169 & 6.101 & 5.247 & 6.104 & 5.402 \\
t(Mean) & 2.539 & -4.556 & 4.679 & -1.484 & -2.847 & -3.553 & 5.417 & 1.616 & 6.065 \\
Sharpe Ratio & 0.370 & 0.665 & 0.682 & 0.216 & 0.415 & 0.518 & 0.790 & 0.236 & 0.885 \\
t against. 0.6 & $\mathbf{- 5 . 2 7 5}$ & 1.386 & 1.764 & $\mathbf{- 9 . 0 0 4}$ & $\mathbf{- 4 . 2 1 0}$ & -1.824 & $\mathbf{3 . 9 4 3}$ & $\mathbf{- 8 . 5 3 2}$ & $\mathbf{5 . 7 3 2}$ \\
\hline
\end{tabular}


Panel C: Hedge portfolio 30\% top and bottom

\begin{tabular}{lcccccccc} 
Month & RetLag1 & Lag2_12 & Lag13_36 & SizeLag1 & Book/Mkt & Profit & AsstGrth & Amihud \\
\hline Mean & -0.957 & 0.535 & -0.502 & -0.732 & -0.904 & 0.486 & 0.013 \\
Std. Dev & 2.654 & 3.494 & 2.529 & 3.150 & 3.434 & 2.088 & 2.007 \\
t(Mean) & $\mathbf{- 8 . 5 6 3}$ & $\mathbf{3 . 6 3 8}$ & $\mathbf{- 4 . 7 1 4}$ & $\mathbf{- 5 . 5 2 2}$ & $\mathbf{- 6 . 2 5 1}$ & $\mathbf{5 . 5 3 0}$ & 0.159 & 2.981 \\
\hline
\end{tabular}

Panel D: Market return plus 30\% top-and-bottom hedge portfolio return

Augmented returns

\begin{tabular}{lccccccccc} 
& \multirow{2}{*}{ Rm-Rf } & \multicolumn{10}{c}{ Augmented returns } \\
\cline { 3 - 10 } & & RetLag1 & Lag2_12 & Lag13_36 & SizeLag1 & Book/Mkt & Profit & AsstGrth & Amihud \\
\hline Mean & 0.490 & -0.467 & 1.025 & -0.012 & -0.242 & -0.414 & 0.976 & 0.504 & 1.050 \\
Std. Dev & 4.584 & 4.528 & 5.618 & 5.502 & 5.517 & 5.039 & 4.820 & 5.630 & 5.112 \\
t(Mean) & 2.539 & -2.448 & 4.335 & -0.052 & -1.043 & -1.950 & 4.810 & 2.124 & 4.880 \\
Sharpe Ratio & 0.370 & 0.357 & 0.632 & 0.008 & 0.152 & 0.284 & 0.702 & 0.310 & 0.712 \\
t against 0.6 & $\mathbf{- 5 . 2 7 5}$ & $\mathbf{- 5 . 5 9 2}$ & 0.701 & $\mathbf{- 1 4 . 0 7 0}$ & $\mathbf{- 1 0 . 5 7 4}$ & $\mathbf{- 7 . 3 4 8}$ & $\mathbf{2 . 1 6 2}$ & $\mathbf{- 6 . 7 3 1}$ & $\mathbf{2 . 3 7 2}$ \\
\hline
\end{tabular}


Table B-9

Cross-correlations between Hedge Portfolios Based on Factors and Characteristic with a Sample Limited by the Inclusion of Amihud's Illiquidity Ratio

Here are correlations between the hedge portfolios corresponding to the nine factors that pass necessary conditions and the hedge portfolios corresponding to the seven characteristics for the restricted sample after including Amihud's illiquidity ratio. The construction of the hedge portfolios is explained in Tables 7 and 8. See the Paper's Appendix for variable descriptions. Correlations that are greater than or equal to 0.5 in absolute value are in boldface.

\begin{tabular}{lcccccccc} 
& Rm-Rf & SMB & HML & RMW & MOM & LT_Rev & DYldCurv & UnexpInf \\
\hline RetLag1 & -0.328 & -0.216 & 0.087 & -0.032 & 0.344 & -0.099 & 0.071 & 0.035 \\
Lag2_12 & -0.098 & -0.139 & -0.060 & -0.046 & $\mathbf{0 . 6 7 8}$ & -0.056 & -0.026 & 0.072 \\
Lag13_36 & 0.084 & -0.101 & -0.243 & -0.049 & 0.040 & -0.361 & -0.013 & 0.060 \\
Size & 0.011 & $\mathbf{- 0 . 7 4 1}$ & -0.110 & -0.006 & 0.289 & -0.069 & 0.001 & 0.466 \\
Book/Mkt & -0.237 & 0.142 & $\mathbf{0 . 5 6 7}$ & 0.171 & -0.247 & -0.014 & -0.218 & -0.129 \\
Profit & -0.148 & -0.437 & 0.261 & $\mathbf{0 . 5 5 5}$ & 0.308 & -0.067 & 0.003 & 0.379 \\
AsstGrth & 0.299 & 0.050 & -0.484 & -0.110 & 0.098 & -0.126 & -0.010 & 0.035 \\
Amihud & -0.169 & $\mathbf{0 . 6 3 9}$ & 0.225 & 0.041 & -0.124 & 0.058 & -0.005 & -0.398 \\
\hline
\end{tabular}

